\title{
Interactions between emotion and action in the brain
}

\author{
Liana Catarina Lima Portugal ${ }^{\mathrm{a}, 2}$, Rita de Cássia Soares Alves ${ }^{\mathrm{a}, 2}$, Orlando Fernandes Junior ${ }^{\mathrm{b}}$, \\ Tiago Arruda Sanchez ${ }^{\mathrm{b}}$, Izabela Mocaiber ${ }^{\mathrm{c}}$, Eliane Volchan ${ }^{\mathrm{d}}$, Fátima Smith Erthal ${ }^{\mathrm{d}}$, Isabel \\ Antunes David ${ }^{\mathrm{a}}$, Jongwan Kim ${ }^{\mathrm{e}}$, Leticia Oliveira ${ }^{\mathrm{a}}$, Srikanth Padmala ${ }^{\mathrm{f}}$, Gang Chen ${ }^{\mathrm{g}}$, \\ Luiz Pessoa $^{\mathrm{e}, \mathrm{h}, 1}$, Mirtes Garcia Pereira ${ }^{\text {a,*,1 }}$
}

${ }^{a}$ Department of Physiology and Pharmacology, Laboratory of Neurophysiology of Behavior, Biomedical Institute, Federal Fluminense University, Niterói, RJ, Brazil

${ }^{\mathrm{b}}$ Laboratory of Neuroimaging and Psychophysiology, Department of Radiology, Faculty of Medicine, Federal University of Rio de Janeiro, Rio de Janeiro, RJ, Brazil

${ }^{\mathrm{c}}$ Laboratory of Cognitive Psychophysiology, Department of Natural Sciences, Institute of Humanities and Health, Federal Fluminense University, Rio das Ostras, RJ, Brazil

${ }^{\mathrm{d}}$ Laboratory of Neurobiology II, Institute of Biophysics Carlos Chagas Filho, Federal University of Rio de Janeiro, Rio de Janeiro, RJ, Brazil

${ }^{\mathrm{e}}$ Department of Psychology, University of Maryland, College Park, MD, USA

${ }^{\mathrm{f}}$ Indian Institute of Science, Bangalore, India

${ }^{g}$ Scientific and Statistical Computing Core, National Institute of Mental Health, USA

${ }^{\mathrm{h}}$ Maryland Neuroimaging Center, University of Maryland, College Park, MD, USA

\section{A R T I C L E I N F O}

\section{Keywords:}

fMRI

Self-relevance

Emotion

Action

Anterior midcingulate cortex

\begin{abstract}
A B S T R A C T
A growing literature supports the existence of interactions between emotion and action in the brain, and the central participation of the anterior midcingulate cortex (aMCC) in this regard. In the present functional magnetic resonance imaging study, we sought to investigate the role of self-relevance during such interactions by varying the context in which threating pictures were presented (with guns pointed towards or away from the observer). Participants performed a simple visual detection task following exposure to such stimuli. Except for voxelwise tests, we adopted a Bayesian analysis framework which evaluated evidence for the hypotheses of interest, given the data, in a continuous fashion. Behaviorally, our results demonstrated a valence by context interaction such that there was a tendency of speeding up responses to targets after viewing threat pictures directed towards the participant. In the brain, interaction patterns that paralleled those observed behaviorally were observed most notably in the middle temporal gyrus, supplementary motor area, precentral gyrus, and anterior insula. In these regions, activity was overall greater during threat conditions relative to neutral ones, and this effect was enhanced in the directed towards context. A valence by context interaction was observed in the aMCC too, where we also observed a correlation (across participants) of evoked responses and reaction time data. Taken together, our study revealed the context-sensitive engagement of motor-related areas during emotional perception, thus supporting the idea that emotion and action interact in important ways in the brain.
\end{abstract}

\section{Introduction}

The processing of emotion-laden information, such as threat, is fast and prioritized. Emotion theories posit that emotions prime organisms for action tendencies (Darwin, 1872; Lang et al., 1997; Damasio, 1999) both in terms of approach and avoidance behaviors (Frijda, 1986). Darwin, for one, argued that emotions are adaptive insofar as they prompt actions that are beneficial to the organism. In line with this notion, manual approach-avoidance tasks with different types of apparatus, using a broad range of affective stimuli, showed that perceiving positive stimuli fosters approach behavior, whereas perceiving negative stimuli facilitates avoidance behavior (Chen and Bargh, 1999; Roelofs et al., 2009; Krieglmeyer and Deutsch, 2010; Saraiva et al., 2013; Phaf et al., 2014). Given the survival-related value of such actions, it is expected that emotional stimuli modulate signals in motor-related areas (Blakemore and Vuilleumier, 2017) much in the same way that they receive

\footnotetext{
* Corresponding author. Federal Fluminense University (UFF) Department of Physiology and PharmacologyHernani Pires de Mello, 101 São Domingos, Niterói, Rio de Janeiro, Cep: 24.210-130, Brazil.

E-mail address: mirtes_pereira@id.uff.br (M.G. Pereira).

1 Joint senior authors.

2 These authors contributed equally to this work.
} 
prioritized visual processing.

Further insight about emotion-motor interactions stems from studies utilizing transcranial magnetic stimulation and/or electromyography, which have reported increased excitability of the corticospinal tract during emotion perception (Oliveri et al., 2003; Hajcak et al., 2007; Coelho et al., 2010; Van Loon et al., 2010; Nogueira-Campos et al., 2014). Furthermore, the emotional valence of a stimulus with which one is about to interact influences motor planning, as captured through the readiness potential, an electrophysiological marker of motor preparation (de Oliveira et al., 2012; Campagnoli et al., 2015). The existence of emotion-action interactions is also supported by functional neuroimaging studies of emotional modulation of motor-related brain areas (de Gelder et al., 2004; Grèzes et al., 2007; Pichon et al., 2008; Pichon et al., 2009; Ahs et al., 2009; Pereira et al., 2010; Pichon et al., 2012; Kveraga et al., 2015; Kolesar et al., 2017; Meyer et al., 2019), even at the level of the spinal cord (Smith and Kornelsen, 2011; McIver et al., 2013; Kornelsen et al., 2014).

More generally, the study of motor repertoires recruited by emotional stimuli has been explored in the non-human animal literature. For example, threat from predators or conspecifics prompts defensive behaviors including overt actions and/or immobility (e.g. Ratner, 1967; Blanchard and Blanchard, 1971). The corresponding human literature is relatively sparse, however. Characterization of defensive reactions in humans has capitalized upon studies of stabilometry, a methodology that assesses whole-body motor reactions (Azevedo et al., 2005; Bastos et al., 2016; Facchinetti et al., 2006; Volchan et al., 2011; for a review see Volchan et al., 2017). The findings from these studies point to similarities between non-human and human defensive behaviors, both of which exhibit motor defensive responses recruited according to threat imminence (Fanselow and Lester, 1988). Particular features of threatening cues and associated context influence how threat imminence is perceived, determining the motor response to be triggered (Blanchard et al., 1986; Volchan et al., 2017). One such property is the self-relevance of the threat context, which can depend on subtle visual changes. For example, modifying the direction from a threat directed away to one directed towards the observer impacts threat perception. In fact, threat stimuli directed towards the observer are perceived as highly threatening, proximal, inescapable, and impossible to hide from (Fernandes et al., 2013; Bastos et al., 2016).

A brain area that is potentially a key site of emotion and motor interactions, and consequently involved in the selection of adaptive responses in different contexts, is the midcingulate cortex (MCC) (Pereira et al., 2010). One of the characteristics of the MCC is its prominent role in skeletomotor control (Vogt and Vogt, 2009), and the area appears to be especially involved with motor patterns that are context dependent (Talairach et al., 1973; Bancaud and Talairach, 1992). The MCC has two divisions, anterior (aMCC) and posterior (pMCC), with distinct functional profiles, cytoarchitecture and connections (for a review see Vogt, 2016). The aMCC has been described in the literature as an import site for cognitive control processes (Shackman et al., 2011), but also activated during fear- and pain-related conditions (Shackman et al., 2011; Vogt, 2016), while the pMCC exhibits almost no emotion-related activity (Vogt, 2005). In a previous study of our group (Pereira et al., 2010), we observed that the aMCC was recruited robustly only when participants performed a motor task in an unpleasant context, reinforcing the idea that the interplay between valence and motor information is important. In the study, aMCC activity during a target detection task paralleled the behavioral modulation associated with negative stimuli, such that increased activity in the negative context was sustained and correlated with the magnitude of the behavioral modulation.

Despite progress in understanding interactions between emotion and motor-related processing, the role of self-relevance during threat perception is poorly understood. In the present study, we addressed this issue using functional magnetic resonance imaging (fMRI) by adopting an experimental approach developed by our group in which behavioral and psychophysiological emotional responses varied as a function of stimulus relevance (Fernandes et al., 2013; Bastos et al., 2016). In these studies, threatening visual stimuli consisted of pictures of a man holding a gun. Self-relevance was increased by changing gun direction from pointing away to pointing directly towards the participant. Here, we investigated brain responses while participants performed a task following exposure to such stimuli. We hypothesized that viewing threat stimuli directed towards the observer would recruit emotional and motor areas more robustly. In particular, some of these areas would exhibit a valence by context interaction, such that the differential response to threat would be enhanced when stimuli were self-relevant.

\section{Methods}

\subsection{Participants}

Forty-nine right-handed undergraduate or graduate students participated in the study ( 26 females, mean age $=26.6$, standard deviation $=$ 5.0). Six participants were excluded from analyses due to excessive errors ( $>25 \%$, mean error rate was $5.5 \%$ ) leading to a final sample of 43 subjects for reaction time data analysis (20 females, mean age $=26.7$, standard deviation $=4.8$ ). Additionally, two volunteers were excluded due to data loss during data transfer, two due to excessive head movement during scanning (greater than $6 \mathrm{~mm}$ ) and one because of poor structural-functional alignment, leading to a final sample of 38 participants (17 females, mean age $=27.1$, standard deviation $=4.8$ ). All participants had normal or corrected-to-normal vision, reported no psychiatric or neurologic problems and were not taking medication with central nervous system action. The project was approved by the local ethics committee of Federal Fluminense University, Rio de Janeiro, Brazil, and each participant gave written informed consent prior to participation.

In a previous study, we reported findings using 26 participants of the sample reported here (Fernandes et al., 2017). However, the study focused on an entirely distinct question, namely it employed machine learning to predict individual-level negative-affect trait measures from brain activation.

\subsection{Stimuli}

Eighty-four pictures were employed. Pictures were either obtained from the World Wide Web, purchased from Getty Images ${ }^{\circledR}$ (http:// www.gettyimages.com), or produced by the authors with support of a professional photographer, except for one picture that was obtained from the International Affective Picture System (IAPS) (Lang et al., 2005). The stimulus size was standardized to $1024 \times 768$ pixels.

The 84 pictures consisted of two sets: threat and neutral stimuli (42 pictures each). Threat stimuli were pictures of a man holding a gun. Neutral stimuli were pictures of a man holding an object, such as a camera or a domestic tool. In both sets, the guns or the neutral objects were either directed towards or away from the participant (21 pictures in each). In all, there were a total of four picture categories: (1) threat stimuli directed towards the participant; (2) threat stimuli directed away from the participant; (3) neutral stimuli directed towards the participant; and (4) neutral stimuli directed away from the participant. The pictures were matched along several properties to minimize potentially confounding effects (Steinmetz et al., 2011). The ethnicity of men holding guns or non-lethal objects was balanced. Threat and neutral stimuli were also matched in terms of physical properties such as brightness, contrast, and spatial frequency (Tables S1 and S2, supplemental material). Electrodermal responses and evaluative characterization of stimuli are depicted in supplemental material, and revealed that our threat manipulation was successful: threat directed towards the participant was judged as more intense, nearer and inescapable, and providing reduced possibility of hiding. Additionally, threat stimuli directed towards the participant induced greater skin conductance response compared to all conditions, indicating an increased recruitment of sympathetic system in 
this context.

\subsection{Experimental design and procedure}

Stimuli were back-projected onto a screen located in front of the participant's body and were viewed inside the scanner using a mirror attached to the head coil. The stimuli were presented using Presentation software (Neurobehavioral Systems, version 11.0, Inc., Albany, CA, USA). The experimental session was divided into four runs. Each run consisted of 14 blocks. The order of blocks of each category (threat stimuli directed towards or away from the participant, and neutral stimuli directed towards or away from the participant) was randomized. Each block consisted of three different pictures ( $5 \mathrm{~s}$ each) of the same category presented in sequence, followed by a 12-s fixation cross. Each trial began with the presentation of the picture $(740 \times 520 \mathrm{~mm})$ together with a central fixation cross $(9 \times 9 \mathrm{~mm})$. Three seconds after picture onset, a square (cue, $35 \times 35 \mathrm{~mm}$ ) appeared around the fixation cross, indicating that the target would appear at any moment. The target, a small central circle (inner circumference diameter of approximately 28 $\mathrm{mm}$ ), appeared 700-1,200 ms after the square, and both remained on until the end of the trial. The fixation cross, the cue, and target were shown over the picture. Participants were instructed to attend to each picture while maintaining their eyes at a fixation spot at the centre of the screen, and to press a button with their right index finger as quickly as possible following target onset. An MR-compatible response key, positioned on the right side of the participant's abdomen, recorded the responses. Each trial during which one picture was shown followed by target detection lasted for $5 \mathrm{~s}$ (Fig. 1). Each run was $378 \mathrm{~s}$ long.

At the beginning of the session, during anatomical scanning, the participants performed a practice task, which was similar to the main experiment except that all images involved pictures of neutral objects, such as tools and furniture, and feedback was displayed on the screen.

\subsection{Image acquisition}

Functional and anatomical MRI data were collected at the Department of Radiology at Hospital Universitário Clementino Fraga Filho (Federal University of Rio de Janeiro) on a 1.5T Siemens (Magnetom Avanto) scanner. The fMRI runs were acquired on a sequential ascending order, using a gradient echo EPI single-shot sequence covering 25 axial slices (4-mm-thick; 0.6-mm gap; TR/TE $=2000 / 40 \mathrm{~ms}$; IST $=80 \mathrm{~ms}$; FOV $=256 \mathrm{~mm}$; matrix, $64 \times 64$; voxel dimensions, $4 \times 4 \times 4.6 \mathrm{~mm}$ ). Head movements were restrained by foam padding. In each run, 198 functional volumes were acquired in a total of four runs. In addition, a three-dimensional high-resolution T1-weighted anatomical image (TR/ $\mathrm{TE}=2730 / 3.27 \mathrm{~ms} ; 128$ slices; 0.6-mm gap; FOV $=250 \mathrm{~mm}$; voxel dimensions $1.33 \times 1 \times 1.33 \mathrm{~mm}$ ) was obtained at the beginning of the session for functional-to-anatomical image registration.

\subsection{Functional MRI preprocessing}

Preprocessing of the functional and anatomical MRI data used AFNI (Cox, 1996; http://afni.nimh.nih.gov/) and the Statistical Parametric Mapping software package (SPM8, Friston et al., 1995; Wellcome Department of Cognitive Neurology, London, UK). The first three volumes of each functional run were discarded to account for equilibration effects. Slice-timing correction used Fourier interpolation (AFNI 3dTshift) to align the onset times of every slice in a volume to the first slice. A six-parameter rigid body transformation (AFNI 3dvolvreg) was used to correct head motion within and between runs by spatially registering each volume to the first volume.

The SPM8 package was used to skull strip the high-resolution anatomical images. Anatomical images were rotated to match the oblique plane of the functional data using AFNI 3dWarp. Each participant's anatomical scan was registered to the TT_N27 template of the AFNI

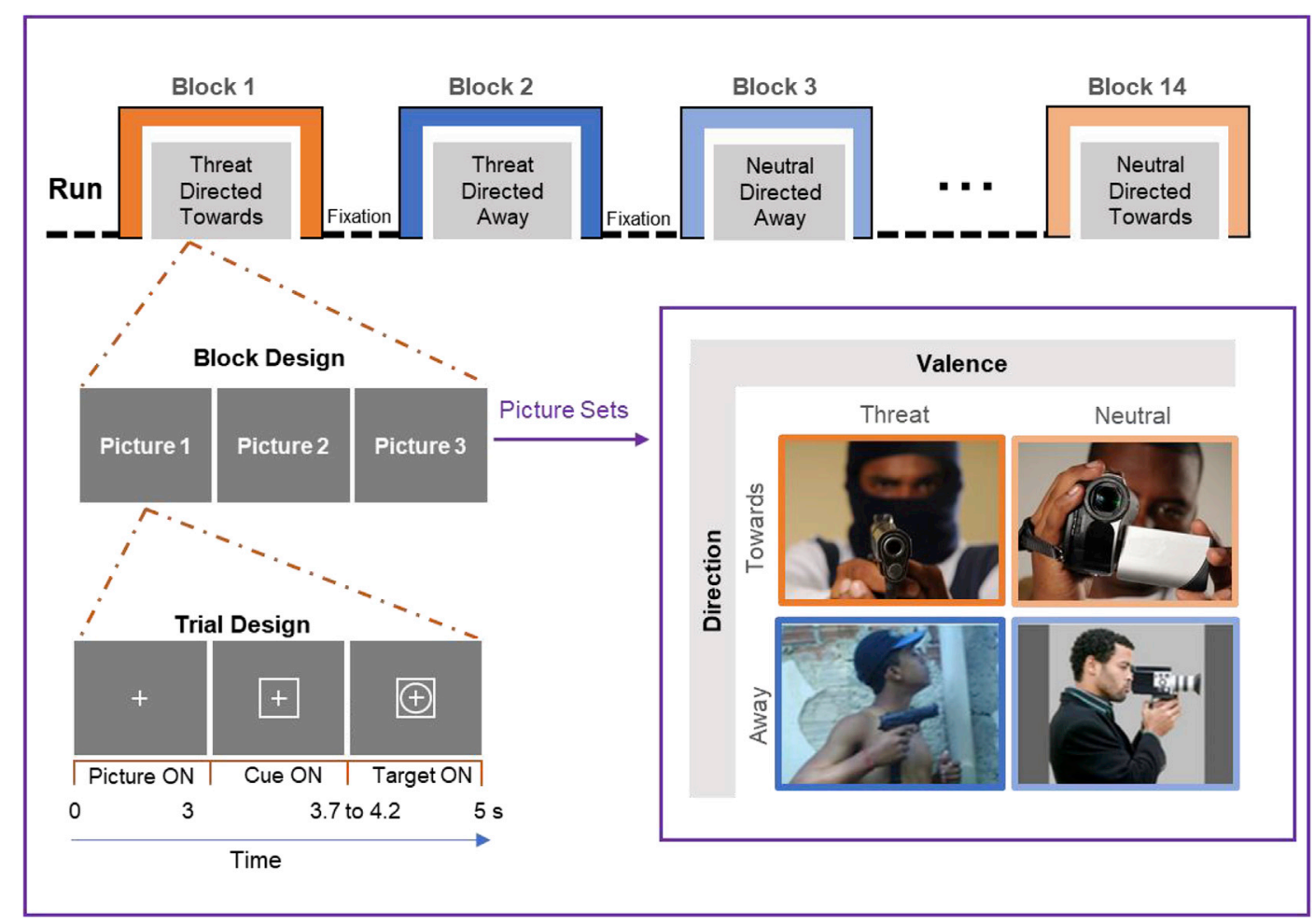

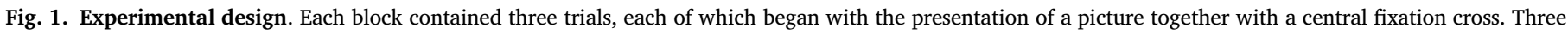

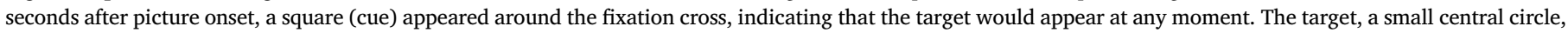

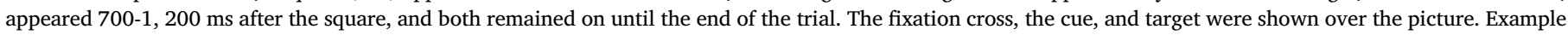

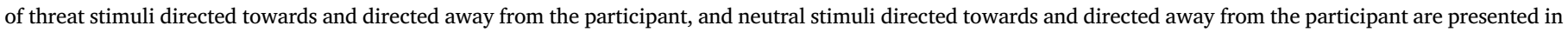
a clockwise fashion. 
package for normalization to Talairach space (Talairach and Tournoux, 1988). The same transformation was applied to the functional data. An 8-mm full-width half-maximum Gaussian filter was used to spatially smooth all volumes, and the average intensity at each voxel (for each run) was scaled to 100 . It should be noted that the use of non-standardized pre-processing pipelines, particularly when utilizing different packages, may contribute to decreased study replicability (Poldrack et al., 2019).

\section{6. fMRI analysis}

Data analysis was performed according to multiple linear regression as implemented in AFNI (using 3dDeconvolve). The first (fixed) level involved determining the regression coefficients of variables of interest, which modelled the effects of each experimental condition: threat stimuli directed towards, threat stimuli directed away, neutral stimuli directed towards, and neutral stimuli directed away. Before estimation via multiple regression, regressors of interest were convolved with a canonical haemodynamic response function (GAM in AFNI; Cohen, 1997). The $2 \mathrm{~s}$ following the cue onset were used as regressors of interest for each condition, and the 12-s fixation cross between blocks was considered as the baseline. The four runs were modelled together. Movement parameters, and their first derivatives, were entered as covariates of no interest associated with the participants' head motion. To further control for head motion, we excluded volumes with a frame-to-frame displacement of more than half of the voxel size from the analysis (Siegel et al., 2014). Only one participant had four volumes excluded.

Second-level group analyses were conducted by means of a repeatedmeasures ANOVA with the factors of valence (threat, neutral) and context (directed towards, directed away). A central goal of the present paper was to test whether the effect of valence was influenced by context, that is, threat direction. Accordingly, the main effect of valence performed in a voxel-wise manner was used to define a focused set of target regions of interest (ROIs) for further testing of an interaction pattern. The main effect of valence was evaluated at a voxel-level threshold of 0.01 (corrected based on False Discovery Rate, FDR). For the 16 ROIs exhibiting a main effect of valence (see Table 1 ), mean regression coefficients for all significant voxels (cluster of activation for the main effect of valence) were extracted for each subject and condition. Each ROI was further interrogated for evidence of a valence by context interaction

Table 1

Main effect of valence (voxelwise analysis). Thresholded at a voxel-level alpha value of 0.01 (FDR corrected).

\begin{tabular}{|c|c|c|c|c|c|}
\hline \multirow[t]{2}{*}{ REGIONS } & \multicolumn{3}{|c|}{$\begin{array}{l}\text { TALAIRACH } \\
\text { COORDINATES }\end{array}$} & \multirow[t]{2}{*}{$\begin{array}{l}\text { CLUSTER } \\
\text { SIZE }\end{array}$} & \multirow[t]{2}{*}{$\mathrm{F}$} \\
\hline & $\mathrm{X}$ & $\mathrm{Y}$ & $\mathrm{Z}$ & & \\
\hline $\begin{array}{l}\text { Right Middle Temporal Gyrus } \\
\text { (EBA) }\end{array}$ & 44 & -67 & -7 & 301 & 37.3 \\
\hline Left Middle Temporal Gyrus (EBA) & -46 & -64 & -1 & 268 & 41.9 \\
\hline $\begin{array}{l}\text { Left Superior Frontal Gyrus } \\
\text { (dmPFC) }\end{array}$ & -4 & 50 & 32 & 228 & 32.8 \\
\hline Left Putamen & -13 & 8 & -1 & 119 & 30.1 \\
\hline Left Lingual Gyrus & -16 & -82 & -13 & 86 & 49.1 \\
\hline Right Putamen/pallidum & 20 & 14 & -4 & 72 & 23.2 \\
\hline Right Supplementary Motor Area & 11 & 8 & 56 & 62 & 36.3 \\
\hline Right Middle Cingulate Cortex & 5 & 17 & 29 & 57 & 32.8 \\
\hline $\begin{array}{l}\text { Right Precentral Gyrus (BA 6/BA } \\
\text { 4) }\end{array}$ & 38 & -7 & 38 & 57 & 28.9 \\
\hline $\begin{array}{l}\text { Right Inferior Frontal Gyrus (p. } \\
\text { triang/vlPFC) }\end{array}$ & 50 & 38 & 8 & 41 & 24.3 \\
\hline $\begin{array}{l}\text { Right Superior Parietal Lobule/ } \\
\text { Precuneus }\end{array}$ & 26 & -49 & 44 & 24 & 22.9 \\
\hline Left Insula & -34 & 26 & 8 & 15 & 18.9 \\
\hline Left Precentral Gyrus (BA 4) & -43 & -7 & 38 & 15 & 20.1 \\
\hline Right Precuneus (BA 7) & 11 & -58 & 47 & 13 & 20.2 \\
\hline Left Cerebellum & -4 & -55 & -37 & 9 & 21.0 \\
\hline Left Amygdala & -25 & -4 & -10 & 8 & 17.4 \\
\hline
\end{tabular}

effect (see section "Region-based Bayesian analysis" below). Finally, for completeness, brain regions with a valence by context interaction effect that survived FDR correction at a voxel-level threshold of 0.05 are reported.

It should be highlighted that, based on the procedure just outlined, functional ROIs were defined using a contrast (main effect of valence) that is statistically orthogonal to the tested effects (interaction term), avoiding bias in the selection (Kriegeskorte et al., 2009)). Note, however, that regions with a crossover interaction pattern (hence, no main effect of valence) would be missed by this method, although potentially they could be identified via the voxel-wise analysis.

Given the potential role of the aMCC for the integration of emotional and motor signals, activity in this region was further investigated using an anatomical atlas-based ROI. The aMCC (anatomical) ROI was created based on the Destrieux et al. (2010) atlas, which subdivides the cingulum into several segments following the antero-posterior direction, as proposed by Vogt (Vogt et al., 2003; Vogt et al., 2006). Mean regression coefficient values, for each subject and condition, were extracted and tested for evidence of a valence by context interaction effect (no thresholding based on functional data was applied).

\subsection{Bayesian statistical analysis}

The null hypothesis significance testing (NHST) framework has come under increased scrutiny in recent years. In particular, the hard threshold of 0.05 has come under attack, with reasonable researchers calling for stricter thresholds (Benjamin et al., 2018) or, conversely, for the dichotomous use of p-values to be abandoned (McShane et al., 2017). We do not consider a binary threshold to be satisfactory and believe that p-values should be treated continuously. In fact, in the present paper, wherever possible, we employed Bayesian statistical analysis (i.e, all analyses except the voxelwise one described above).

As Bayesian analysis is not as widely used, we briefly compare this framework to NHST. Consider a scenario in which a single one-sample $t$ test with 20 degrees of freedom (e.g., 21 subjects) is employed in the NHST setting. The null hypothesis $\mathrm{H}_{0}$ is that the population mean is zero. Suppose that the data indicate that $\mathrm{t}_{20}=2.85$. If $\mathrm{H}_{0}$ were true, the probability of observing a $t_{20}$-value so large is rather low (0.01 in Fig. 2, left). The $t_{20}$-value thus provides a measure of "surprise": How surprising would it be to observe such an extreme value in a world in which $\mathrm{H}_{0}$ were really true? The extent of surprise corresponds, of course, to $\mathrm{P}\left(\right.$ data $\mid \mathrm{H}_{0}$ ). By custom if $\mathrm{P}<0.05$, then one declares that the effect is "statistically significant".

The Bayesian framework aims to answer a different, though, related question: what is the probability of a research hypothesis $\mathrm{H}$ given the data, $\mathrm{P}(\mathrm{H} \mid$ data $)$ ? Note what is being "measured" and what is the "given" in this proposition, as opposed to the preceding NHST formulation (i.e., $\mathrm{P}\left(\right.$ data $\left.\mid \mathrm{H}_{0}\right)$ ). Such a probability can be computed by using Bayes' rule (for an introductory text, see Kruschke, 2010). In a typical setting, the research hypothesis is a statement that refers to an effect or parameter being estimated (e.g., mean, difference of means, etc.). An attractive property of this framework is that it is not typically formulated to generate a binary decision ("real effect" vs "noise", or "significant" vs. "not significant") but instead to obtain the entire probability density distribution associated with $\mathrm{P}(\theta \mid$ data), where $\theta$ is the parameter being estimated (Fig. 2, right). This so-called posterior distribution is interpreted in a natural way, although it may take getting used to for those who are unfamiliar with Bayesian inferences. For example, $\mathrm{P}(\theta>0$ | data), which we call $\mathrm{P}+$, is the area under the curve in Fig. 2 (right); in the present case, $\mathrm{P}+=0.99$, namely, there is very strong evidence that the effect of interest (e.g., mean, difference of means, etc.) is greater than zero (conditional on the data, the prior distribution, and the model). Note that small values of $\mathrm{P}+$ convey support for a negative effect; for example, $\mathrm{P}+=0.01$ indicates that the probability of the effect being positive is only 0.01 , which implies that the probability of it being negative is 0.99 . Finally, the estimation of posterior distributions requires the 

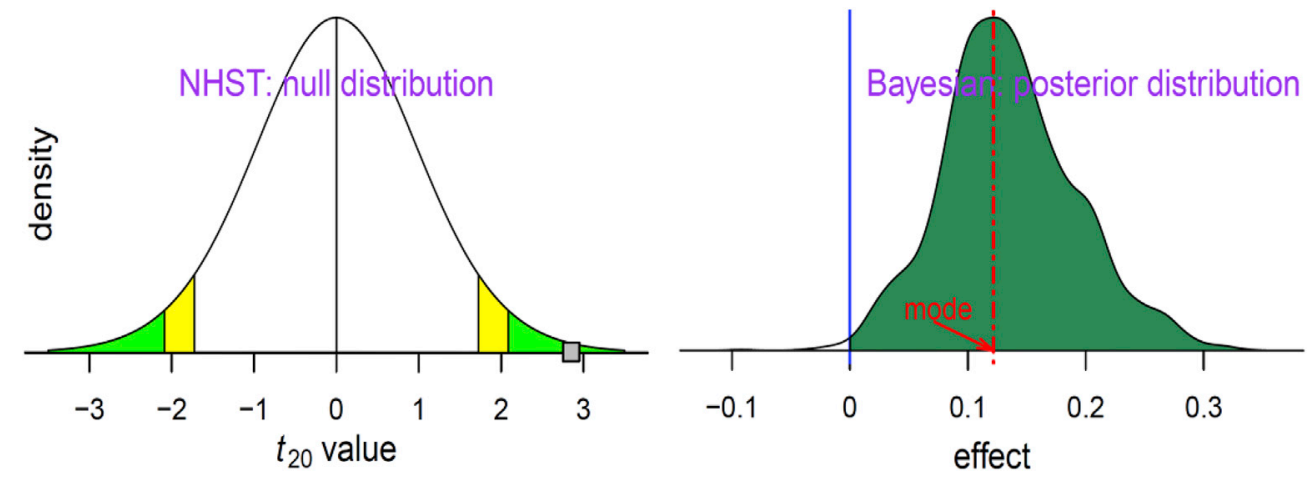

Fig. 2. Different probability definitions and goals of conventional and Bayesian frameworks. Left: Statistical inferences under null hypothesis significance testing are based on how extreme the data are in the context of the null hypothesis. Green/yellow tails symbolize a two-sided significance level of $0.05 / 0.1$. In the example, the data produces a t-value of 2.85 (small gray square). Right: Inferences under the Bayesian framework directly address the question of research interest: what is the probability of the effect magnitude being greater than 0 with the data at hand? The "output" in a Bayesian inference comprises the entire posterior density function, which summarizes the uncertainty in estimating the effect magnitude. specification of a prior distribution. In all analyses reported here, we employed so-called "uninformative" priors, which accordingly do not have a notable impact on the conclusions.

The posterior distribution provides a summary description of the likelihood of observing parameter values given the data, so it naturally conveys variability. Some authors use cut-off points to summarize "strong", "moderate", or "weak" evidence, but we encourage an approach that both quantifies and qualifies the evidence, without making decisions in terms of "passes threshold" versus "fails to pass threshold". Note that we do not employ Bayes factors, which some have advocated as a potential feature of Bayesian modeling. Because Bayes factors consider the probability of "null" effects (e.g., a mean of zero) versus an "alternative" effect (e.g., a mean different from zero), we believe it is also problematic, because formulating the problem in terms of "null" effects of exactly zero is often unrealistic (because effects of experimental manipulations are seldom zero), thus largely inflating the evidence for the alternative hypothesis (thus creating "large" Bayes factors). See Chen et al. (2019a) for further discussion. Finally, given that we do not view thresholding as adequate, the $\mathrm{P}+$ probability values that we provide are (by definition) "one-sided". For readers who absolutely insist on comparing P+ values with standard cut-offs that are "two-sided", they should bear in mind our definition.

\subsection{Bayesian analysis of reaction time and individual ROIs}

Bayesian analyses were performed via the brms $\mathrm{R}$ package (Bürkner, 2017), which employs the Stan probabilistic language to compute posterior distributions via state-of-the-art Monte Carlo Markov Chain modelling (Stan Development Team, 2016). To analyze the effects of valence, context, and their interaction, we ran three separate Bayesian analyses: the two main effects and the interaction. If we consider the four levels of the experimental variables (in order: threat_towards, neutral_towards, threat_away, neutral_away), the effect of valence can be obtained via the contrast expression $(+1-1+1-1)$, the effect of context via the contrast expression $(+1+1-1-1)$, and their interaction via the contrast $(+1-1-1+1)$. Given this coding, the three models were simply of the form

effect $\sim \operatorname{Normal}(\alpha, \sigma)$

$\sigma \sim \operatorname{Normal}(0,10)$

where " " indicates "distributed as", $\alpha$ is a single intercept, $\sigma$ is the residual variation, and the last line specifies the prior distribution.

\subsection{Region-based Bayesian analysis}

A standard ROI analysis evaluates the effect of interest for each ROI, separately. Given the multiplicity of ROIs, investigators commonly perform some correction for multiple comparisons, say via Bonferroni correction. Here, we analyzed the interaction between valence and context by performing a Bayesian multilevel analysis of the ROI data by using the Region-Based Analysis (RBA) program of the AFNI suite (Chen et al., 2019b). In this approach, the data from all ROIs are included in a single multilevel model that evaluates the effects of interest. By doing so, the contributions to fMRI signals of subject-level effects (i.e., subject effect across conditions), and ROI-level effects (i.e., ROI effect across subjects), can be accounted for in a model that simultaneously ascertains the interaction effect.

Thus, under the standard general linear model, the effect at each region is estimated independently from other regions; there is no information shared across regions, hence, the multiple comparisons step. In contrast, in the Bayesian multilevel framework the effects across regions are shared (technically, via partial pooling) by assuming that the ROI contributions are normally distributed. The latter implies a Gaussian prior distribution on the ROI effects. The "output" of the Bayesian multilevel model comprises only one overall posterior that is formulated as a joint distribution in a high-dimensional parameter space (thus, no "correction" is needed). For summary purposes, posteriors of the effects for every ROI can be plotted separately; but they are not independent and technically are simply marginal distributions (that is, projections along particular variables). For formal details of the approach adopted here, please refer to Chen et al. (2019b); for a less technical exposition, see Chen et al. (2019a).

\subsection{Brain-behavior correlation}

To test whether the magnitude of the emotional modulation in the aMCC (anatomical ROI) was related to the magnitude of the behavioral emotional modulation, we used a modulation index for both measures. In the case of behavior, the index was defined as the mean RT to targets during threat trials minus the mean RT to targets during neutral trials, for the directed towards and directed away contexts, separately. Accordingly, negative values of the index indicated that participants were faster to detect the target when threat stimuli were presented; positive values indicated the reverse. In the case of aMCC responses, we computed an analogous index based on the average regression coefficient values for each condition of interest.

We performed Bayesian analysis of the brain-behavior relationship for the right and left aMCC (anatomical), separately, by using the brms R/ Stan package. Because this involved a linear relationship, the model was simply

$\operatorname{aMCC} \sim \operatorname{Normal}(\mu, \sigma)$

$\mu \sim \alpha+\beta \mathrm{xRT}$

$\alpha \sim \operatorname{Normal}(0,10)$ 
$\beta \sim \operatorname{Normal}(0,10)$

$\sigma \sim$ HalfCauchy(10)

where the second line specifies the linear association, and the last three indicate the prior distributions. The estimates of the slope $(\beta)$ were contrasted between conditions (the two different contexts) by comparing their difference in a Bayesian manner (again by using the brms R/Stan package). Additionally, we employed the same approach to conduct exploratory analyses in regions showing some evidence of an interaction between valence and context (specifically, a $\mathrm{P}+$ of approximately 0.95 ).

\section{Results}

The analyses reported next were all performed within a Bayesian framework, except the voxelwise analyses which followed the standard statistical approach employed in neuroimaging. Here, we report the extent of evidence, $\mathrm{P}(\theta>0 \mid$ data), as $\mathrm{P}+$. Values of $\mathrm{P}+$ closer to 1 indicate stronger evidence that the effect of interest (e.g., mean, difference of means, etc.) is greater than zero (see Methods). Small values of $\mathrm{P}+$ convey the extent of support that the effect is negative; that is, the probability that the effect is negative is given by $(1-\mathrm{P}+)$.

\subsection{Behavioural performance}

Behaviorally, we observed a tendency for the speeding up of RT to targets when viewing negative pictures $(467 \mathrm{~ms}$ ) relative to those following neutral pictures (474 ms) during directed towards trials (effect size $d=-0.39$ ). During directed away trials, mean RTs were the same (474 $\mathrm{ms}$ ) for threat and neutral trials (effect size $\mathrm{d}=-0.02$ ). The Bayesian analysis of RTs revealed evidence for a valence by context interaction $(\mathrm{P}+=0.040)$, an effect of valence $(\mathrm{P}+=0.038)$, and an effect of context $(\mathrm{P}+=0.051)$. Fig. 3 shows the RT data together with the Bayesian posterior density plots. In all three cases, because most of the evidence for the effect of interest is negative, the $\mathrm{P}+$ values are close to zero.

\subsection{Brain responses}

\subsubsection{Main effect of valence: voxelwise analysis}

Evidence for a main effect of valence was detected bilaterally in occipitotemporal regions linked to visual processing, right cingulate and peri-cingulate cortex, left insula, right parietal and bilaterally in frontal areas (Table 1 and Fig. 4.). Subcortically, valence effects were detected in the left cerebellum, left amygdala, and bilateral putamen. For completeness, the main effect of context is reported in Table 2.

\subsubsection{Valence by context interaction: voxelwise analysis}

Although our goal was to investigate interaction effects in ROIs exhibiting evidence of a valence effect, for completeness we also performed a voxelwise analysis of the interaction, which was detected in visual areas (bilateral inferior/middle occipital gyrus, bilateral lingual gyrus, left inferior temporal/occipital gyrus, right middle occipital/ temporal gyrus), as well as left inferior frontal gyrus (pars Opercularis),

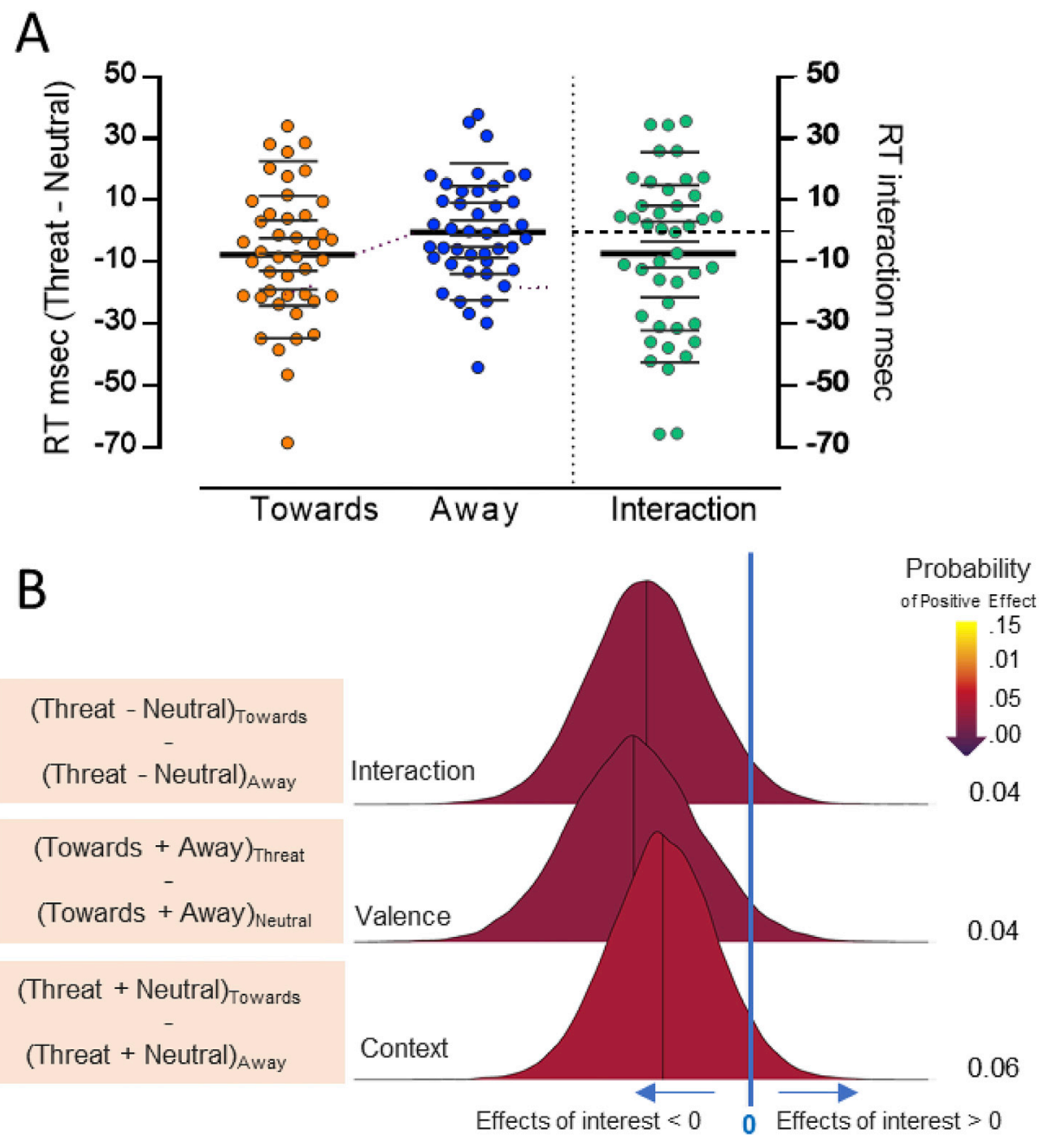

Fig. 3. Behavioral data and Bayesian posterior density plots.

(A) The circles show reaction time (RT, ms) differences (threat minus neutral), and a behavioral interaction index (RT difference during directed towards minus RT difference during directed away). Thicker lines mark the mean and thinner lines the deciles for each experimental condition. The thin purple line marks the shift between means. Data illustrations used some of the graphical tools proposed by Rousselet et al. (Rousselet et al., 2017). (B) Bayesian posterior density plots of the valence by context interaction effect, the main effect of valence, and the main effect of context for RT data. Inferences under the Bayesian framework directly address the probability of the effect magnitude being greater than 0 , which we call $\mathrm{P}+$. The color bar represents $\mathrm{P}+$. Note that the reddish to dark purple color associated with small values of $\mathrm{P}+$ convey support that the effect is negative; in this case, $\mathrm{P}+=0.04$ indicates that the probability of the effect being positive is only 0.04 , so that the probability of it being negative is 0.96 . 


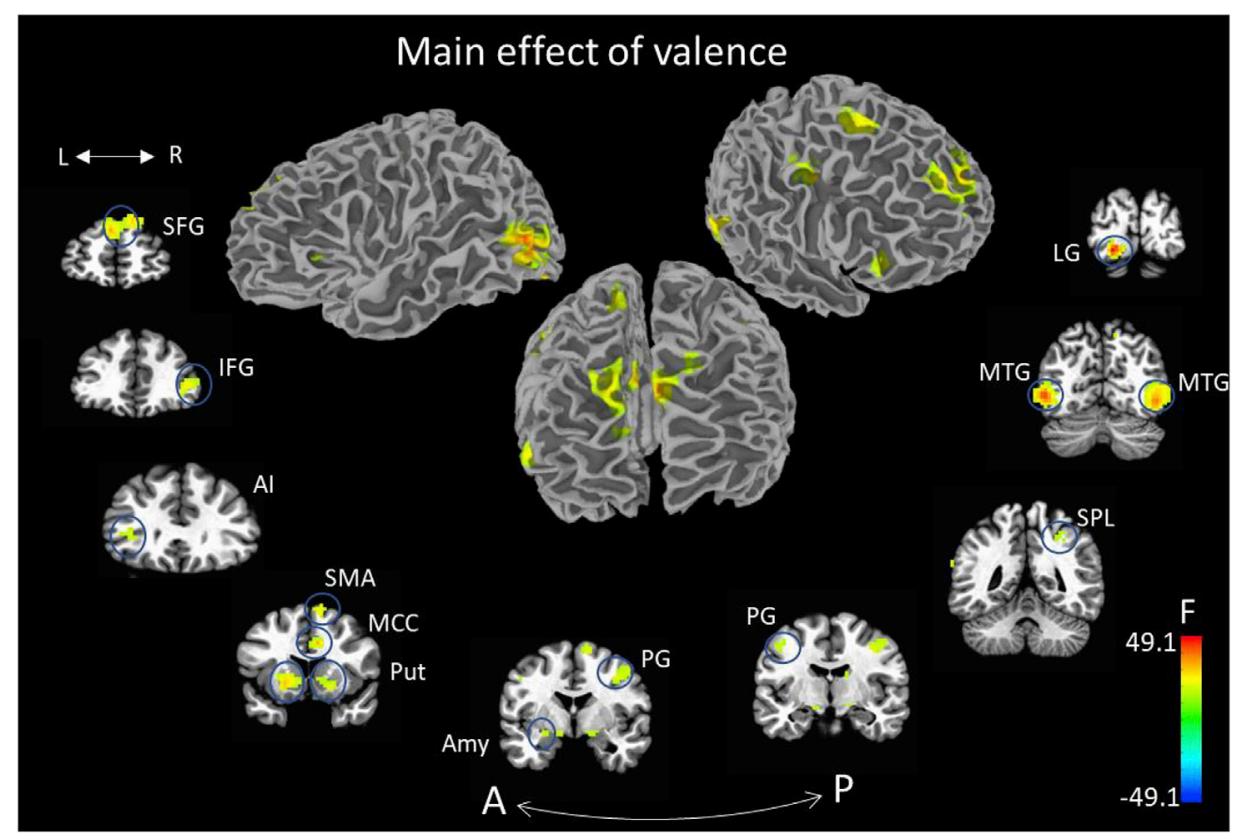

Fig. 4. Voxelwise analysis: Main effect of valence. See Table 1 for the Tailarach coordinates. The color bar represents the value of the F-statistic. Abbreviations: A: Anterior; AI: Anterior insula; Amy: Amygdala; IFG: Inferior frontal gyrus; L: Left; LG: Lingual gyrus; MCC: Middle cingulated cortex; MTG: Middle temporal gyrus; P: Posterior; PG: Precentral gyrus; Put: Putamen; R: Right; SFG: Superior frontal gyrus; SMA: Supplementary motor area; SPL: Superior parietal lobule.

Table 2

Main effect of context (voxelwise analysis). Thresholded at a voxel-level alpha value of 0.01 (FDR corrected).

\begin{tabular}{|c|c|c|c|c|c|}
\hline \multirow{2}{*}{$\begin{array}{l}\text { REGIONS } \\
\text { MAIN EFFECT OF CONTEXT }\end{array}$} & \multicolumn{3}{|c|}{$\begin{array}{l}\text { TALAIRACH } \\
\text { COORDINATES }\end{array}$} & \multirow{2}{*}{$\begin{array}{l}\text { CLUSTER } \\
\text { SIZE }\end{array}$} & \multirow[t]{2}{*}{$\mathrm{F}$} \\
\hline & $\mathrm{X}$ & $\mathrm{Y}$ & $\mathrm{Z}$ & & \\
\hline Right Parahippocampal Gyrus & 26 & -43 & -7 & 3595 & 150.7 \\
\hline Right Lingual Gyrus & 26 & -88 & -4 & 512 & 107.3 \\
\hline Right Superior Parietal Lobule & 26 & -58 & 53 & 416 & 36.5 \\
\hline Left Fusiform Gyrus & -37 & -76 & -13 & 369 & 74.9 \\
\hline $\begin{array}{l}\text { Right Middle Temporal Gyrus } \\
\text { (EBA) }\end{array}$ & 41 & -67 & 17 & 276 & 79.4 \\
\hline Left Superior Parietal Lobule & -28 & -52 & 47 & 100 & 20.9 \\
\hline Left Precuneus & -1 & -55 & 38 & 74 & 23.4 \\
\hline Left Parahippocampal Gyrus & -13 & -10 & -19 & 8 & 15.6 \\
\hline
\end{tabular}

and right supplementary motor area (Table 3 and Fig. 5).

3.2.3. Interaction between valence and context: Region of interest analysis

As stated, as our focus was to investigate whether or not threat direction influenced emotional modulation, interactions between valence and context were further investigated in ROIs selected based on the main effect of valence (Table 1). In all regions exhibiting evidence for a main effect of valence, activity was greater during threat conditions relative to neutral ones (except for the lingual gyrus, in which the reverse was observed).

We performed a Bayesian multilevel analysis that included all ROIs simultaneously in a single model (Methods). The posterior distributions for the valence by context effect are shown in Fig. 6, where the color indicates $\mathrm{P}+$, the probability of the effect being greater than zero. For example, strong evidence was determined for the middle temporal gyrus bilaterally (at sites labelled in the literature as the "extrastriate body area"), the right supplementary motor area (SMA), the left anterior insula, and the right precentral gyrus. Fig. 7 shows the mean parameter estimate for each subject per condition for the ROIs exhibiting strong evidence of a valence by context interaction. But note that since the Bayesian framework is not dependent on a target false-positive rate (say, 0.05), we can consider all effect strengths in a continuous fashion. For
Table 3

Valence by context interaction (voxelwise analysis). Thresholded at a voxellevel alpha value of 0.05 (FDR corrected).

\begin{tabular}{|c|c|c|c|c|c|}
\hline \multirow[t]{2}{*}{$\begin{array}{l}\text { REGIONS } \\
\text { Valence by Context Interaction }\end{array}$} & \multicolumn{3}{|c|}{$\begin{array}{l}\text { TALAIRACH } \\
\text { COORDINATES }\end{array}$} & \multirow[t]{2}{*}{$\begin{array}{l}\text { CLUSTER } \\
\text { SIZE }\end{array}$} & \multirow[t]{2}{*}{$\mathrm{F}$} \\
\hline & $\mathrm{x}$ & $\mathrm{Y}$ & $\mathrm{Z}$ & & \\
\hline $\begin{array}{l}\text { Right Inferior/Middle Occipital } \\
\text { Gyrus }\end{array}$ & 44 & -68 & -9 & 131 & 35.3 \\
\hline Right Lingual Gyrus & 11 & -80 & -6 & 44 & 31.4 \\
\hline Left Lingual Gyrus & -10 & -83 & -3 & 39 & 39.4 \\
\hline $\begin{array}{l}\text { Left Inferior/Middle Occipital } \\
\text { Gyrus }\end{array}$ & -40 & -80 & -6 & 35 & 21.7 \\
\hline $\begin{array}{l}\text { Left inferior Frontal } \\
\text { Gyrus(Opercularis) }\end{array}$ & -49 & 19 & 6 & 20 & 22.4 \\
\hline Right Supplementary Motor Area & 2 & 10 & 54 & 15 & 18.7 \\
\hline Left Inferior Temporal Gyrus & -40 & -50 & -15 & 13 & 21.3 \\
\hline Right Superior Occipital Gyrus & 29 & -83 & 30 & 6 & 17.8 \\
\hline
\end{tabular}

further evaluation of the interaction effects, Table 4 reports the effect sizes (Cohen's d) of the contrast between threat and neutral for each context.

\subsubsection{Response pattern in the anterior MCC}

We analyzed responses in terms of valence, context, and their interaction in the aMCC based on anatomical atlas-based ROIs. Bayesian analysis revealed strong evidence for the interaction on the left $(\mathrm{P}+=$ 0.975), and good support on the right $(\mathrm{P}+=0.941)$. Both right and left were rather robustly driven by valence (left: $\mathrm{P}+=0.998$; right: $\mathrm{P}+=$ 0.998), but not by context (left: $\mathrm{P}+=0.449$; right: $\mathrm{P}+=0.580$ ). See Fig. 8B for bayesian posterior density plots of interaction, valence and context effects and Fig. 8C for mean parameter estimate for each subject per condition.

\subsubsection{Brain-behavior correlations}

A previous study by our group showed that aMCC activity paralleled behavioral emotional modulation in an aversive context (Pereira et al., 2010). Accordingly, we employed a behavioral modulation index defined as the mean RT to targets during threat trials minus the mean RT to targets during neutral trials, separately for the directed towards and 


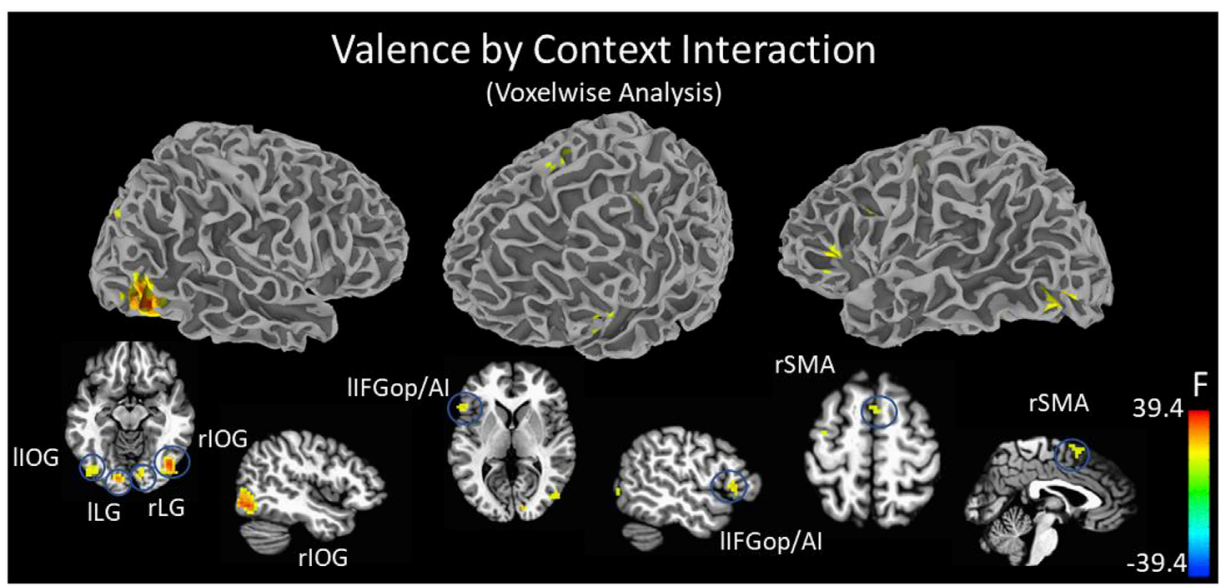

Fig. 5. Voxelwise analysis: Valence by context interaction effect. See Table 3 for the Tailarach coordinates. The color bar represents the value of the F-statistic. Abbreviations: IFGop/AI: Inferior frontal gyrus pars opercularis/anterior insula; IOG: Inferior occipital gyrus; L: Left; LG: Lingual gyrus; R: Right; SFG: Superior frontal gyrus; SMA: Supplementary motor area.

\section{Valence by Context Interaction ROI analysis}

Right Middle Temporal Gyrus

Left Middle Temporal Gyrus

Right Supplementary Motor Area

Left Anterior Insula

Right PreCentral Gyrus

Right PreCuneus

Right Superior Parietal Lobule

Right Anterior Middle Cingulate Cortex

Left PreCentral Gyrus

Right Inferior Frontal Gyrus

Left Putamen

Right Putamen/Pallidum

Left Superior Frontal Gyrus

Left Cerebellum

Left Amygdala

Left Lingual Gyrus

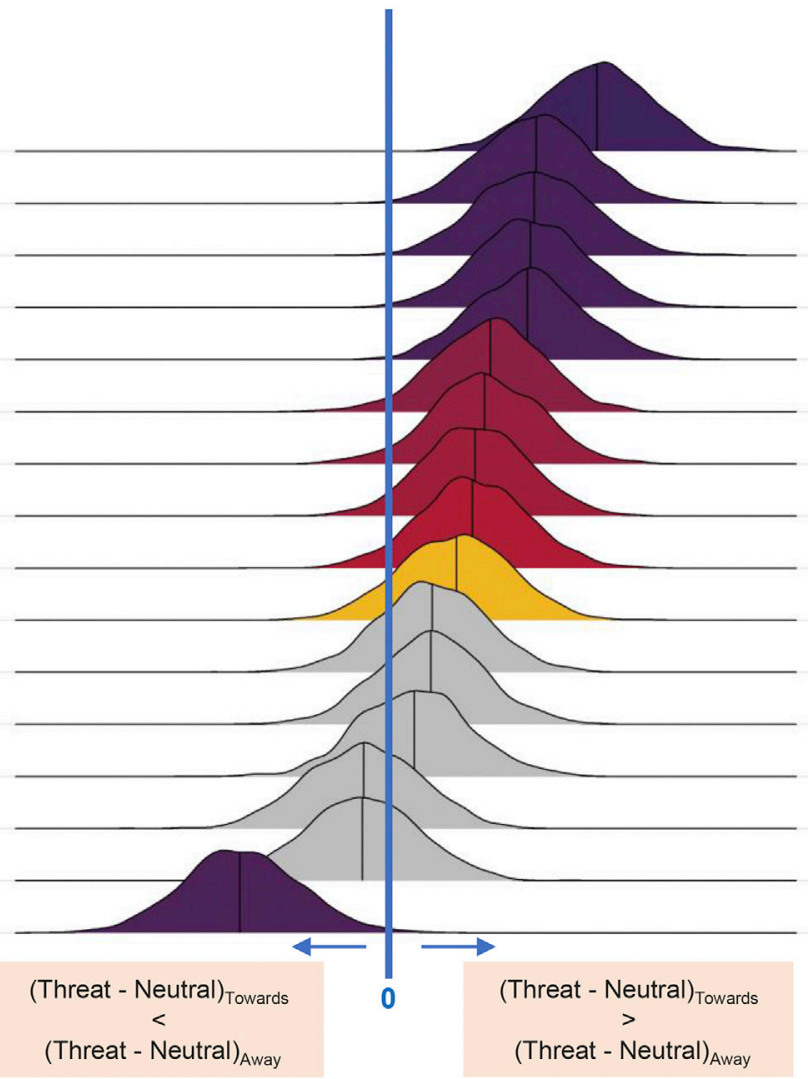

Probability of Positive Effect

ช 1.00

.95

1

1

.15

.01

$1 \quad .05$

0.96

0.95

0.95

0.94

0.88

0.78

0.78

0.66

0.33

0.31

0.01

Fig. 6. Bayesian posterior density plots of the valence by context interaction for ROIs reported in Table 1 . The color bar indicates $\mathrm{P}+$, the probability of the effect being greater than zero. Larger values of $\mathrm{P}+$ convey support that the activity was greater for $(\text { Threat - Neutral) })_{\text {Towards }}$ relative to $(\text { Threat }- \text { Neutral })_{\text {Away; }}$; small values of $\mathrm{P}+$ convey support of the reverse pattern. The color bar represents $\mathrm{P}+$. See the caption of Fig. 3 for discussion of interpretation.

directed away contexts. Corresponding indices were defined for aMCC (anatomical) activity, and a linear association between the two was evaluated. Fig. 8 A illustrates the left and right anatomical aMCC ROI.

For the directed towards context, we observed evidence for a negative relationship between behaviour and brain responses (Fig. 8D and E, top): faster RTs for threat relative to neutral stimuli were associated with increased aMCC responses (threat vs. neutral). For the directed towards context, evidence was strong for the right $(\mathrm{P}+=0.029$; Spearman $\mathrm{rho}=$
$-0.47)$ and especially the left $(\mathrm{P}+=0.007$; Spearman rho $=-0.39)$ hemisphere. For the directed away context, evidence of a relationship between brain and behavior was not particularly noteworthy (left: $\mathrm{P}+=$ 0.810; Spearman rho $=0.17$; right: $\mathrm{P}+=0.643$; Spearman $\mathrm{rho}=0.11$ ). A Bayesian direct comparison of the association between the two contexts (towards vs. away) revealed strong support only for the right hemisphere (left: $\mathrm{P}+=0.212$; right: $\mathrm{P}+=0.026$ ).

We employed the same approach above to conduct exploratory 

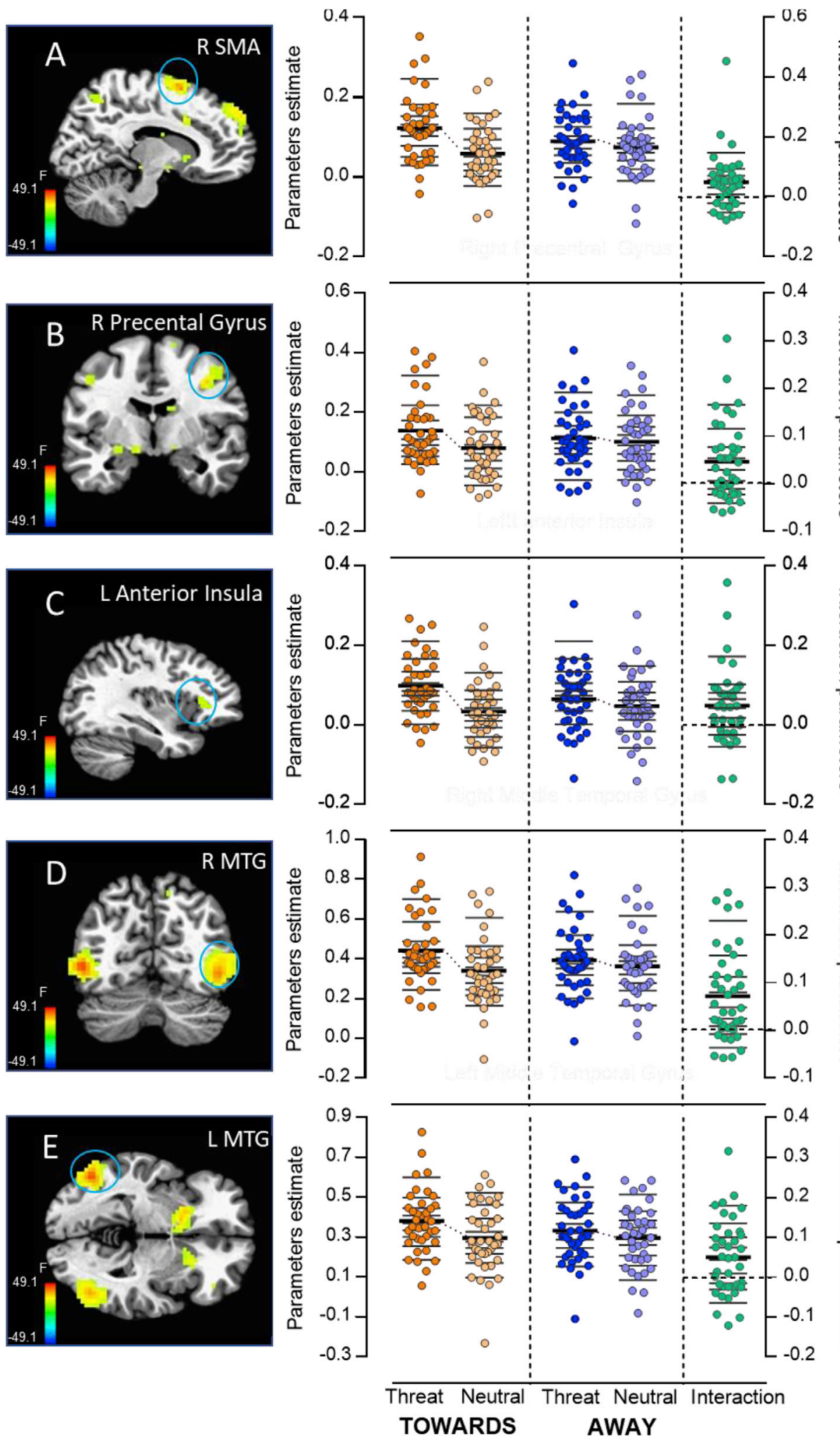

Fig. 7. Regions of interest and valence by context interaction. (A) Supplementary Motor Area $(11,8,56)$ (B) Precentral Gyrus $(38,-7,38)$ (C) Left Anterior Insula $(-34,26,8)$ (D) Right Middle temporal Gyrus (MTG, Right: 44, -67, -7) (E) Left Middle temporal Gyrus (MTG, -46, -64, $-1)$. Circles represent the mean parameter estimate for each subject per condition: Threat ${ }_{\text {Towards, Neu- }}$ tral $_{\text {Towards, }}$ Threat ${ }_{\text {Away }}$, Neutral $_{\text {Away }}$. The interaction index was calculated as follows: ((Threat - Neutral) $)_{\text {Towards }}$ - (Threat - Neutral) $)_{\text {Away }}$ ). Thicker lines mark the mean and thinner lines the deciles for each experimental condition. The thin purple line marks the shift between means. Data illustrations used some of the graphical tools proposed by Rousselet et al. (Rousselet et al., 2017).

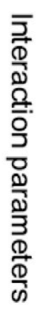

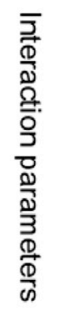

analyses in regions showing some evidence of an interaction between valence and context ("some evidence" was defined as a $\mathrm{P}+$ of approximately 0.95$)$. Based on the posterior distributions in Fig. 6, we considered the following ROIs to investigate brain-behavior correlations: right and left middle temporal gyrus, right supplementary motor area, left anterior insula, right precentral gyrus, right precuneus, right superior parietal lobule and right aMCC (the latter was originally defined functionally based on the effect of valence; Fig. 4). Posterior distributions for brain-behavior correlations for towards and away contexts and for the difference of the correlations between contexts are shown in Fig. 9. In the directed towards context, evidence of a brain behaviour correlation was especially strong in right aMCC (functional) but also evident in right SMA, left anterior insula, and right precentral gyrus. For the directed away context, brain-behaviour associations did not receive support from 
Table 4

Effect size (Cohen's D) of the difference between threat and neutral valence for each context and for the interaction. Talairach coordinates for each ROI are reported in Table 1.

\begin{tabular}{|c|c|c|c|}
\hline REGIONS OF INTEREST & $\begin{array}{l}\text { Towards } \\
\text { Threat-Neutral }\end{array}$ & $\begin{array}{l}\text { Away } \\
\text { Threat- } \\
\text { Neutral }\end{array}$ & $\begin{array}{l}\text { Towards (Threat- } \\
\text { Neutral) - } \\
\text { Away (Threat- } \\
\text { Neutral) }\end{array}$ \\
\hline $\begin{array}{l}\text { Right Middle Temporal Gyrus } \\
\text { (EBA) }\end{array}$ & 1.16 & 0.35 & 0.75 \\
\hline $\begin{array}{l}\text { Left Middle Temporal Gyrus } \\
\text { (EBA) }\end{array}$ & 1.05 & 0.48 & 0.51 \\
\hline $\begin{array}{l}\text { Left Superior Frontal Gyrus } \\
\text { (dmPFC) }\end{array}$ & 0.66 & 0.56 & 0.02 \\
\hline Left Putamen & 0.82 & 0.52 & 0.13 \\
\hline Left Lingual Gyrus & -1.01 & -0.68 & -0.46 \\
\hline Right Putamen/pallidum & 0.80 & 0.53 & 0.13 \\
\hline $\begin{array}{l}\text { Right Supplementary Motor } \\
\text { Area }\end{array}$ & 0.85 & 0.28 & 0.53 \\
\hline $\begin{array}{l}\text { Right Middle Cingulate } \\
\text { Cortex }\end{array}$ & 0.73 & 0.37 & 0.26 \\
\hline $\begin{array}{l}\text { Right Precentral Gyrus (BA } \\
\text { 6/BA 4) }\end{array}$ & 0.90 & 0.19 & 0.56 \\
\hline $\begin{array}{l}\text { Right Inferior Frontal Gyrus } \\
\text { (p. triang/vlPFC) }\end{array}$ & 0.69 & 0.57 & 0.20 \\
\hline $\begin{array}{l}\text { Right Superior Parietal } \\
\text { Lobule/Precuneus }\end{array}$ & 0.89 & 0.25 & 0.38 \\
\hline Left Insula & 0.94 & 0.21 & 0.50 \\
\hline Left Precentral Gyrus (BA 4) & 0.83 & 0.29 & 0.33 \\
\hline Right Precuneus (BA 7) & 0.76 & 0.23 & 0.43 \\
\hline Left Cerebellum & 0.37 & 0.47 & -0.09 \\
\hline Left Amygdala & 0.47 & 0.53 & -0.16 \\
\hline
\end{tabular}

the data. For the explicit Bayesian comparisons of correlations between towards and away contexts, there was weak support for the right aMCC (functional), right SMA, and left insula. For completeness, Table 5 summarizes effect sizes (rho values) of the brain-behavior correlations for each ROI and context.

\section{Discussion}

In the present study, we sought to investigate how emotional processing is affected by context. In particular, we hypothesized that selfrelevance would influence both behavior and associated brain responses. Self-relevance was manipulated by having participants view pictures containing guns that were directed at or away from them. We identified a group of brain areas where activity increased during threat relative to neutral conditions, with a subset of them (occipital cortex, precentral gyrus, supplementary motor area, and insula) exhibiting stronger evidence of a valence by context effect. Finally, we observed a brain-behavior correlation in the anatomically defined right aMCC, which robustly differed between contexts.

One of the features of the present work was that, wherever possible, it followed a Bayesian data analysis approach. Accordingly, we did not consider probability values dichotomously (say, "significant" if less than 0.05 ) but as providing continuous evidence for support for the hypothesis of interest, given the data. Furthermore, we adopted wide, poorly informative prior distributions that had very little discernible impact on our conclusions.

\subsection{Regions modulated by threat relevance}

We observed robust valence by context interactions in regions involved in motor-related processing. Specifically, in both the right SMA and the right precentral gyrus, the differential response to threat vs. neutral was enhanced when the context was more relevant to the participant (directed towards condition). Whereas an increase in activation of cortical motor-related areas while participants observe negative stimuli has been reported (de Gelder et al., 2004; Grèzes et al., 2007;
Pichon et al., 2008; Pichon et al., 2009; Ahs et al., 2009; Pereira et al., 2010; Van den Stock et al., 2011; Conty et al., 2012; Pichon et al., 2012; Kveraga et al., 2015), the role of self-relevance remains poorly understood. Grèzes et al. (2013) reported that both the SMA and the precentral gyrus were engaged preferentially to body expressions of anger oriented to self when compared to anger oriented to other, and suggested that the recruitment of these areas might be related to the need of selecting specific behavioural strategies when one is the potential target of someone's anger.

Conty et al. (2012) combined fMRI and electroencephalography in humans, and obtained evidence that, $200 \mathrm{~ms}$ after stimulus onset, the premotor cortex integrated gaze, gesture, and emotion. They suggested that the early binding of visual social signals displayed by an agent engaged the dorsal pathway and the premotor cortex, possibly to facilitate the preparation of an adaptive response to another person's immediate intention. We thus suggest that, in the context of our experiment, an increase in threat relevance impacts motor-related processing, possibly to implement an appropriate defensive response.

Another region that revealed a robust interaction effect between valence and context was in the middle temporal gyrus, at a location corresponding to the extrastriate body area, or EBA (Downing et al., 2001; Urgesi et al., 2004). Middle temporal gyrus activity was increased for threat stimuli in both contexts but the impact was greater when stimuli were directed towards the participant. The EBA site in the middle temporal gyrus has also been shown to be engaged by emotion-laden stimuli (Grosbras and Paus, 2006; Ponseti et al., 2006; Grèzes et al., 2007; Peelen et al., 2007; Pichon et al., 2008; Flaisch et al., 2009; Sinke et al., 2010; Kret et al., 2011; Kveraga et al., 2015; Van den Stock et al., 2015). It has been proposed that the function of this area goes beyond the mere perception of body shape, as it might provide an interface between perceptual and motor processes (Astafiev et al., 2004; David et al., 2007; Kuhn et al., 2011; Tomasino et al., 2012; Limanowski et al., 2014; Orgs et al., 2016; Simos et al., 2017). For example, Zimmermann et al. (2017) suggested that the middle temporal gyrus/EBA interacts more strongly with dorsal-stream regions, when compared to other portions of the occipito-temporal cortex involved in processing body parts and object identification, and proposed that the area contributes to planning goal-directed actions.

\subsection{Anterior midcingulate cortex and behavioral modulation}

We previously suggested that the aMCC is a site of interaction of negative valence and motor-related signals (Pereira et al., 2010), which motivated the examination here of self-relevance by using an anatomically based ROI. Our results revealed increased aMCC anatomically defined activity for threat vs. neutral stimuli in both directed towards and directed away threat contexts, consistent with an extensive literature pointing to the engagement of the aMCC in aversive processing (for a review see Vogt, 2005).

There has been growing interest in considering the midcingulate cortex as a unique cingulate region with a particular functional profile (Vogt 2016). Initial models of the anterior cingulate cortex suggested that rostral ACC and aMCC (also called dorsal ACC in some studies) specialize according to emotional and cognitive processes, respectively (Bush et al., 2000). In subsequent work, the aMCC has been proposed to be involved in emotion-, cognition-, and pain-related processing (Shackman et al., 2011); see also Misra and Coombes (2015). In addition, meta-analysis studies have proposed that the aMCC plays a central, integrative role in emotion regulation (Kohn et al., 2014), and is part of a core system for implementing self-control across emotion and action domains (Langner et al., 2018). In our previous study, the aMCC was recruited robustly only when participants performed a task in negative contexts, and responses mirrored the pattern of behavioral modulation associated with negative stimuli (Pereira et al., 2010). In the present study, we found a correlation between aMCC activity and behavior during the self-relevant threat context, whereby faster reaction times for threat (vs. neutral) stimuli 

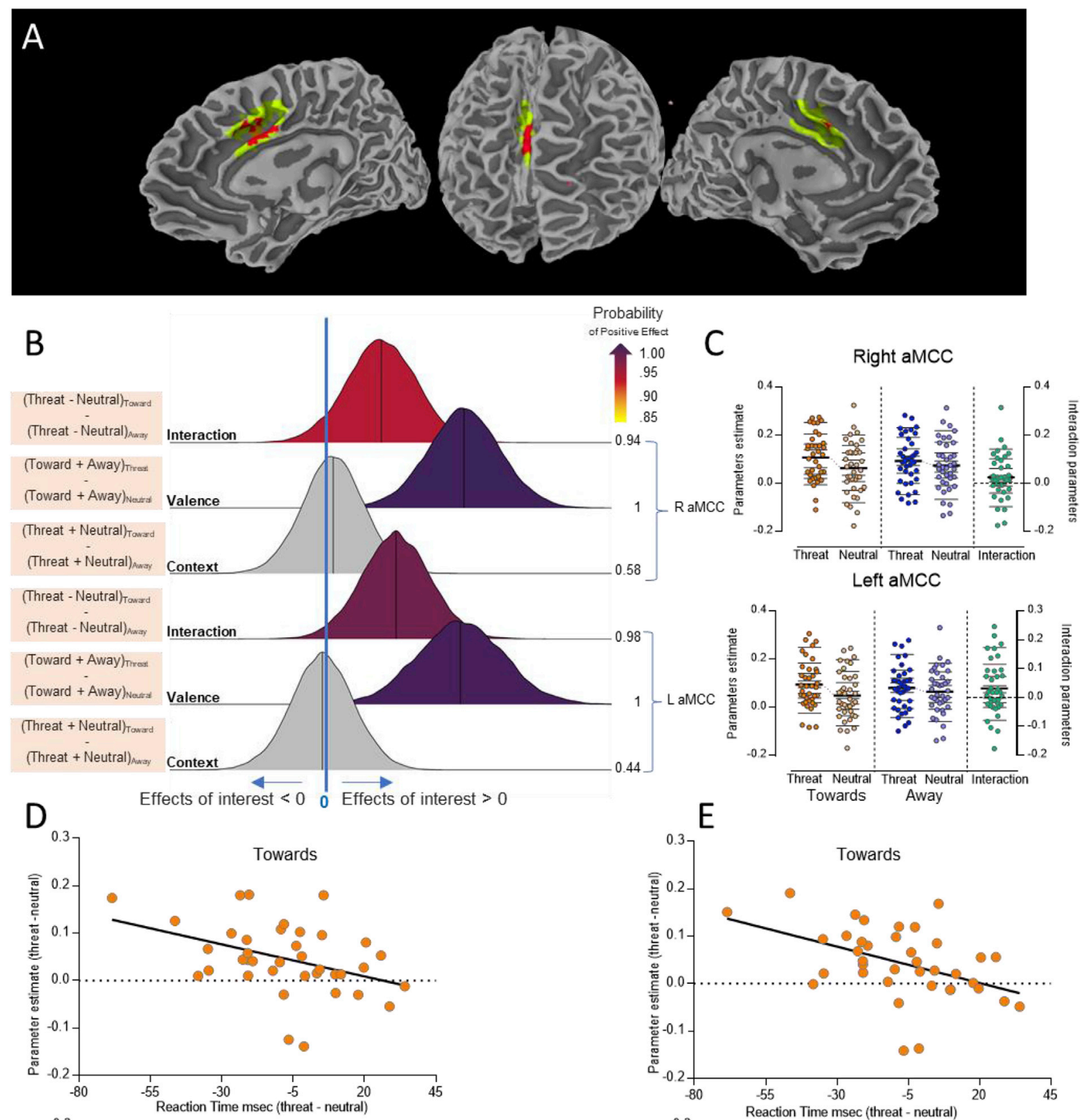

$\mathrm{E}$
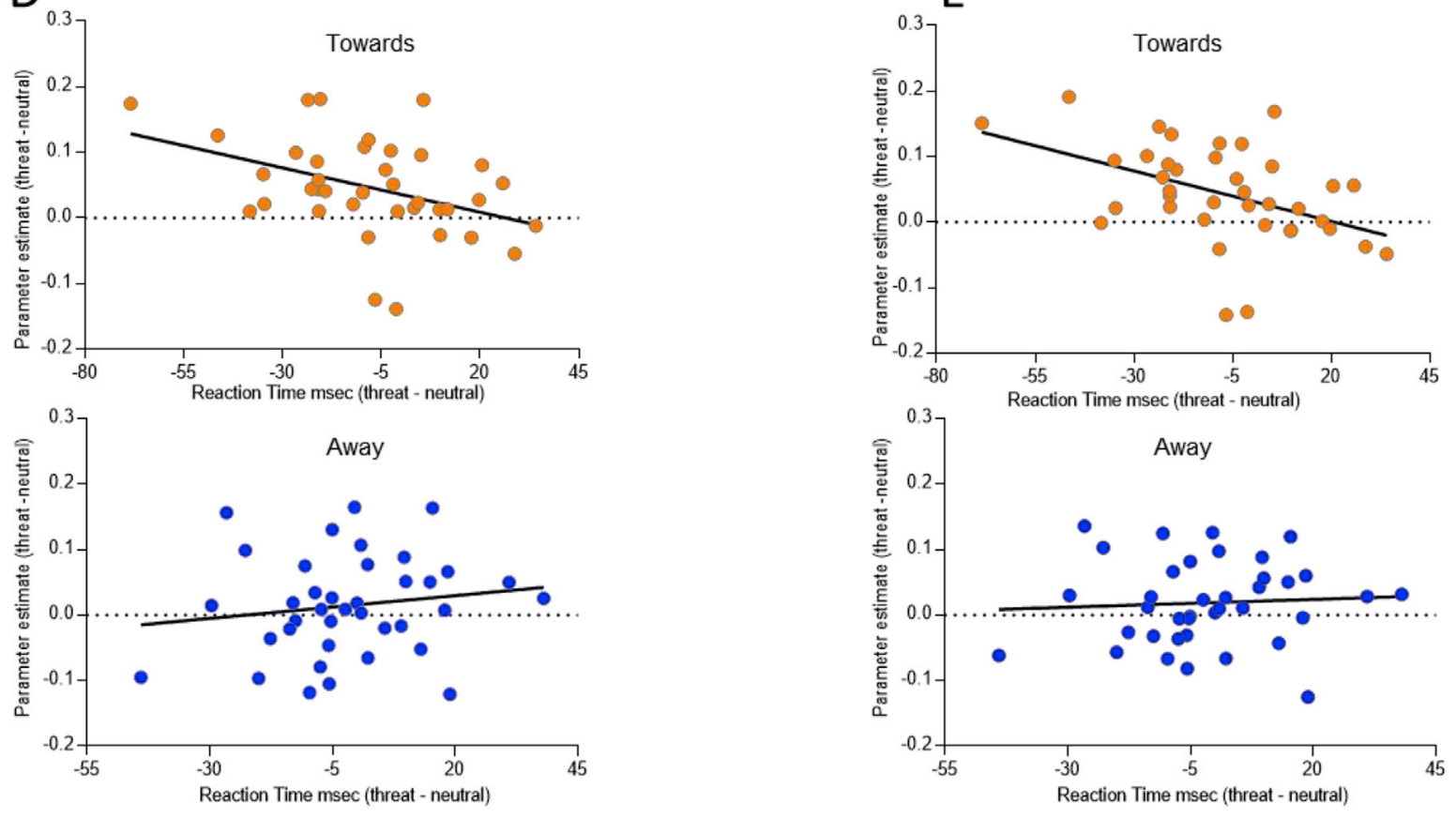

Right aMCC

Fig. 8. Anterior middle cingulate cortex (aMCC). (A) The anatomical ROI (green) is shown together with the midcingulate cluster that exhibited a main effect of valence (orange); the overlap between the two is shown in red. (B) Bayesian posterior density plots of interaction $\left((\text { Threat - Neutral })_{\text {Towards }}-(\text { Threat-Neutral })_{\mathrm{Away}}\right)$, valence $\left((\text { Towards }+ \text { Away })_{\text {Threat }}-(\text { Towards }+ \text { Away })_{\text {Neutral }}\right)$ and context $\left((\text { Threat }+ \text { Neutral })_{\text {Towards }}-(\text { Threat }+ \text { Neutral })_{\text {Away }}\right)$ effect for anterior right and left middle cingulate ROI based on atlas-based anatomical ROIs. The color bar indicates $\mathrm{P}+$, the probability of the effect being greater than zero. The color bar represents $\mathrm{P}+$. See the caption of Fig. 3 for discussion of interpretation. (C) Parameter estimates for the anatomical aMCC ROI. (see Fig. 7 for additional definitions). (D-E) Brain-behavior correlations in the directed towards (top) and in the directed away context (bottom) for the left (D) and right (E) aMCC. 


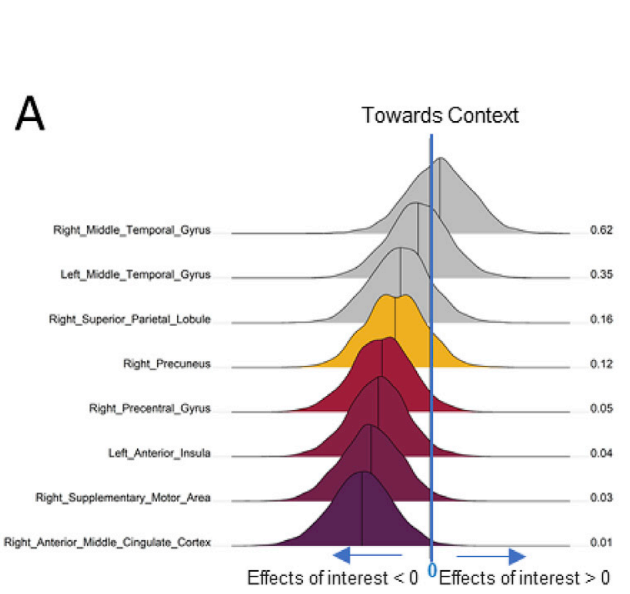

\section{Brain Behavior Correlations} Exploratory Analyses

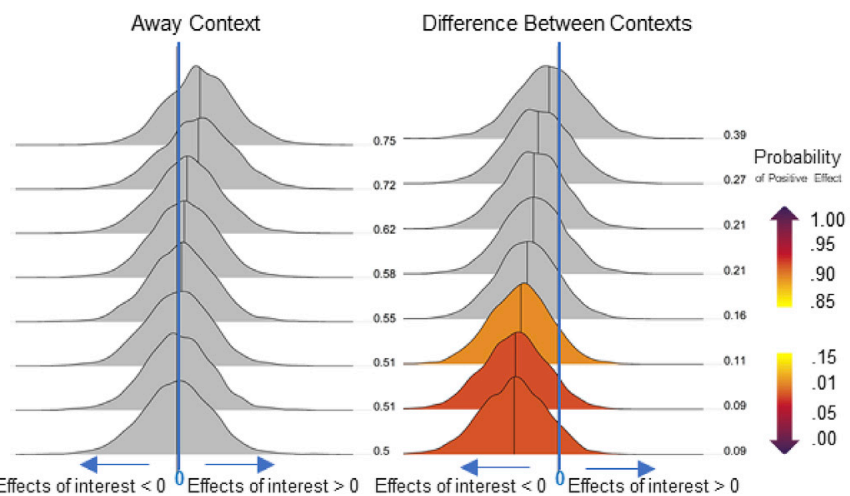

B

\section{Towards}
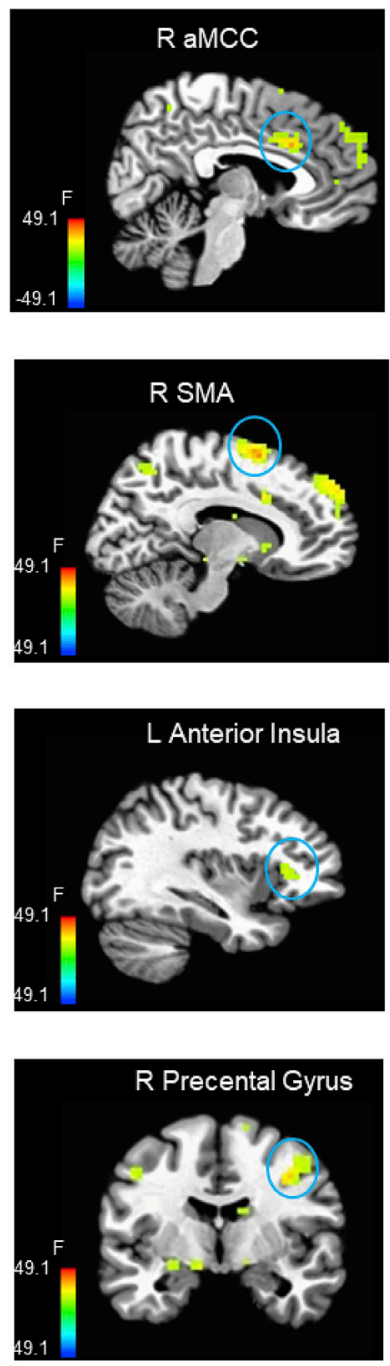
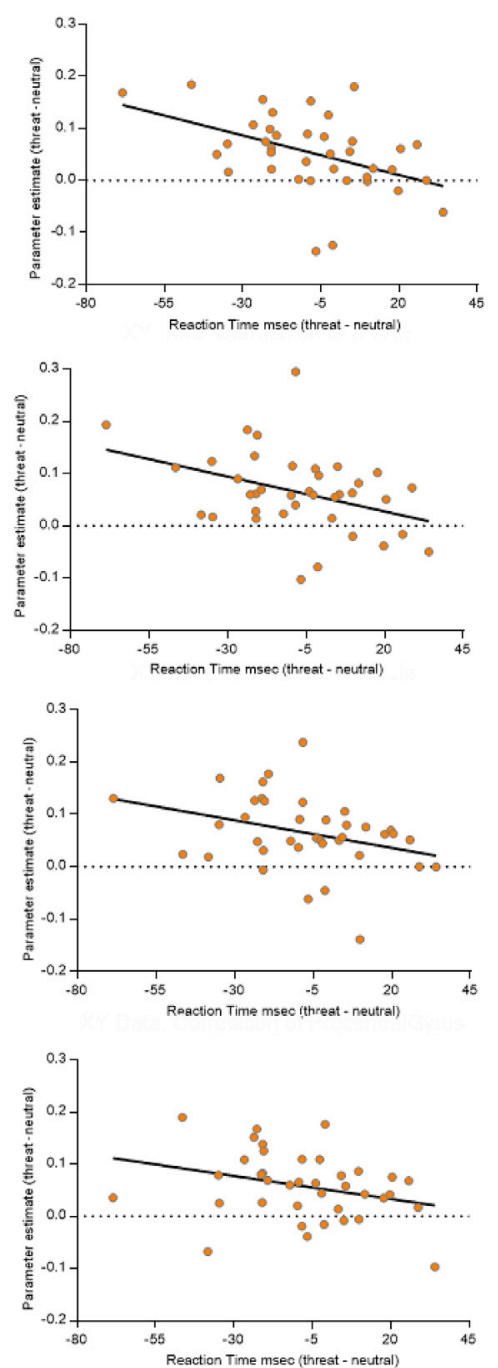

Away
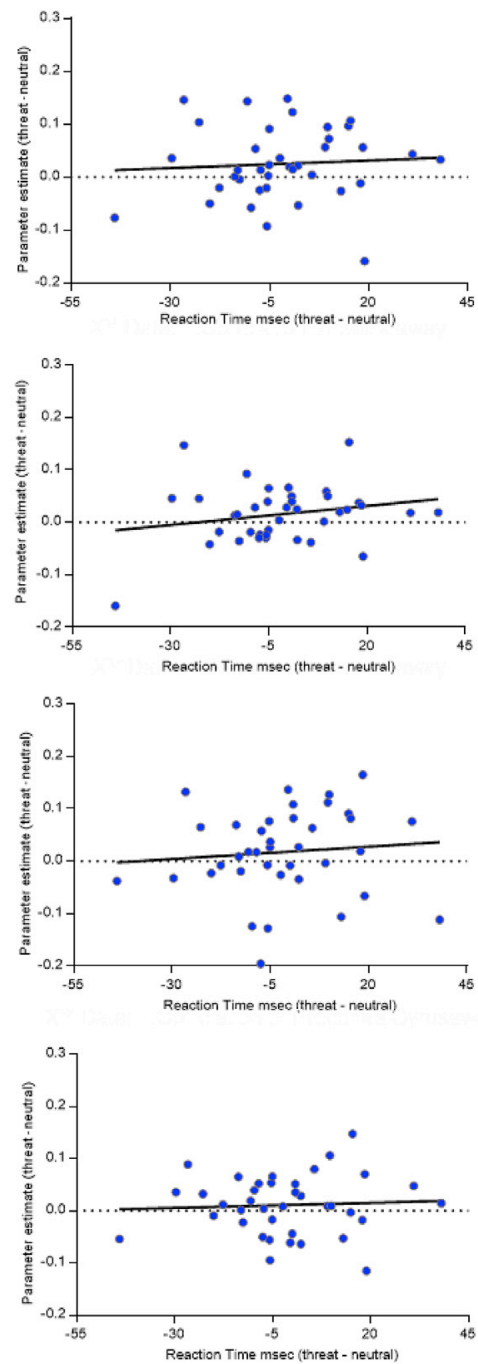

Fig. 9. Brain-behavior relationship in regions showing evidence of an interaction between valence and context. (A) Bayesian posterior density plots of the brain-behavior correlation for the towards context, the away context, and the difference between them. The color bar indicates $\mathrm{P}+$, the probability of the effect being greater than zero. See the caption of Fig. 3 for discussion of interpretation. (B) Brain-behavior correlation in the directed towards (left plots) and in the directed away contexts (right plots) for the right anterior middle cingulate cortex (R aMCC, functionally defined), right supplementary motor area (rSMA), left anterior insula and right precentral gyrus. 
Table 5

Brain-behavior correlations for regions of interest with evidence of a valence by context interaction effect.

\begin{tabular}{lll}
\hline & TOWARDS CONTEX & $\begin{array}{l}\text { AWAY } \\
\text { CONTEXT }\end{array}$ \\
\hline REGIONS OF INTEREST & Rho & Rho \\
Right Middle Temporal Gyrus & 0.17 & 0.08 \\
Left Middle Temporal Gyrus & 0.06 & 0.24 \\
Right Supplementary Motor Area & -0.34 & 0.14 \\
Left Insula & -0.31 & 0.21 \\
Right Precentral Gyrus & -0.32 & 0.02 \\
Right Precuneus & -0.16 & 0.10 \\
Right Superior Parietal Lobule/Precuneus & -0.14 & -0.01 \\
Right Anterior Midcingulate cortex & -0.46 & 0.14 \\
\hline
\end{tabular}

were associated with increased activity in the aMCC (threat vs. safe). In other words, participants that exhibited greater speeding-up in RT for threat also exhibited increased threat-related brain responses. We also note that the reduction in RT for threat observed in the present study is in line with our previous observation that participants were quicker to perform a task in the presence of threat directed towards the self (Fernandes et al., 2013).

We conjecture that participants with greater recruitment of midcingulate cortex exhibited an enhanced implementation of active defensive responses. In line with this possibility, Straube et al. (2009) found activation in the aMCC to be relevant when executive functions are more strongly necessary, such as in situations of high vigilance and action prompting. The present results also converge with the proposal that the aMCC is an important node for emotion and motor interaction in the service of adaptive responses to threat (Pereira et al., 2010; Shackman et al., 2011).

We note that a brain-behavior correlation analogous to that found for the aMCC was observed in the right SMA, precentral gyrus, and anterior insula. In this context, it is relevant that neurons in the aMCC (specifically in the rostral cingulate zone) project to other motor-related areas, including the premotor and supplementary motor cortices (Picard and Strick, 1996, see Shackman et al., 2011 for a review). These brain-behavior correlations revealed in the aMCC (anatomically defined) a stronger relationship between evoked brain responses and target detection times during the self-relevant condition relative to the directed-away context (via a direct comparison of the two conditions). In this context, and as noted above, Langner et al. (2018) proposed that the MCC, medial premotor regions, and anterior insula are essential for implementing self-control across emotion and action. In addition, a recent meta-analysis uncovered a potential association between the integrity of these regions and diverse psychiatric syndromes, including mood and anxiety disorders (Goodkind et al., 2015).

\subsection{Brain areas responding to threat}

Of the areas exhibiting a main effect of valence, we would like to draw attention to the amygdala, a region that has been exhaustively studied in the context of negative processing. This region did not exhibit signs of a valence by context interaction; it did not seem to be affected by increasing threat relevance to the participant. This finding is relevant in the context of the debate of the nature of amygdala responses, namely the extent to which it is modulated by high-level factors such as attention and context, for example. Whereas we have reported that the amygdala is modulated by attention and contextual information (Pessoa, 2013), amygdala responses in the present study were not robustly sensitive to self-relevance. The present results illustrate how the amygdala functions, in some instances, in a more "basic" and less integrative manner.

\section{Limitations}

Some limitations of the present work deserve discussion. We did not observe clear support for a valence by context interaction pattern in the RT data. Whereas we observed a reduction in RT when performing a task in the directed towards context in our previous behavioral study (Fernandes et al., 2013), evidence for this modulation effect was modest here. This difference is possibly due to the smaller sample size used in the current fMRI study, which also compromises the statistical power to detect such interaction effects in a voxelwise analysis. In addition, scanning was performed at $1.5 \mathrm{~T}$, which has reduced sensitivity compared to $3 \mathrm{~T}$ systems. In fact, we only detected a small amygdala cluster (8 voxels) with evidence of a main effect of emotion.

A potential limitation of our study is related to the analysis selection procedure, which focused on ROIs based on the main effect of valence. As our study aimed to understand the effect of context on the processing of emotion-laden images, we felt that this choice was defensible. However, although the main effect of valence is statistically orthogonal to the interaction, given that ROI selection and subsequent testing were based on the same dataset, the procedure is not ideal from the independence standpoint; anatomically defined ROIs or those based on previous published work would have been preferable (Kriegeskorte et al., 2009). In addition, as brain-behavior correlations were investigated at the ROI level, this might have distorted the findings somewhat given that an ROI is treated as a homogenous entity.

\section{Conclusions}

How does the brain integrate emotion-related information and context? In the present study, we manipulated the self-relevance of threat stimuli by manipulating the direction of threat (guns pointed towards or away from the self). Using a Bayesian analysis framework, we identified multiple brain regions sensitive to threat direction, including the middle temporal gyrus/EBA, SMA, and precentral gyrus. We believe the contextsensitive recruitment of motor-related areas (SMA and precentral gyrus) is particularly noteworthy as they provide clues as to how emotion and action signals are integrated. In addition, the present results strengthen the idea that the aMCC is an important node for emotion and motor interaction, as responses in this brain region were correlated with behavior. More broadly, in the same manner that emotion interacts with perception and cognition, it is likely to interact with motor functions in the service of adaptive behaviors.

\section{Declaration of competing interest}

None.

\section{CRediT authorship contribution statement}

Liana Catarina Lima Portugal: Conceptualization, Formal analysis, Investigation, Writing - original draft, Visualization. Rita de Cássia Soares Alves: Conceptualization, Formal analysis, Investigation, Writing - original draft, Visualization. Orlando Fernandes Junior: Conceptualization, Writing - original draft, Visualization, Data curation, Investigation. Tiago Arruda Sanchez: Conceptualization, Visualization, Data curation, Project administration, Investigation. Izabela Mocaiber: Conceptualization, Writing - original draft, Writing - review \& editing. Eliane Volchan: Conceptualization, Writing - original draft, Writing review \& editing, Project administration. Fátima Smith Erthal: Conceptualization, Writing - original draft, Writing - review \& editing. Isabel Antunes David: Conceptualization, Writing - original draft, Writing - review \& editing. Jongwan Kim: Visualization, Formal analysis, Resources. Leticia Oliveira: Conceptualization, Formal analysis, Investigation, Writing - original draft, Visualization, Project administration, Funding acquisition. Srikanth Padmala: Visualization, Formal analysis, Resources. Gang Chen: Visualization, Resources, Writing - review \& editing, Software. Luiz Pessoa: Writing - original draft, Conceptualization, Writing - review \& editing, Formal analysis, Resources, Visualization, Supervision, Software. Mirtes Garcia Pereira: 
Conceptualization, Formal analysis, Investigation, Writing - original draft, Writing - review \& editing, Visualization, Project administration, Supervision, Funding acquisition.

\section{Acknowledgments}

This work was supported in part by federal and state Brazilian research agencies (CAPES 001, CAPES/PRINT, CNPq and FAPERJ). Luiz Pessoa received support from the National Institute of Mental Health (R01 MH071589).

\section{Appendix A. Supplementary data}

Supplementary data to this article can be found online at https://doi. org/10.1016/j.neuroimage.2020.116728.

\section{References}

Ahs, F., Pissiota, A., Michelgård, A., Frans, O., Furmark, T., Appel, L., Fredrikson, M., 2009. Disentangling the web of fear: amygdala reactivity and functional connectivity in spider and snake phobia. Psychiatr. Res. 172, 103-108. https://doi.org/10.1016/ j.pscychresns.2008.11.004.

Astafiev, S.V., Stanley, C.M., Shulman, G.L., Corbetta, M., 2004. Extrastriate body area in human occipital cortex responds to the performance of motor actions. Nat. Neurosci. 7, 542-548. https://doi.org/10.1038/nn1241.

Azevedo, T.M., Volchan, E., Imbiriba, L.A., Rodrigues, E.C., Oliveira, J.M., Oliveira, L.F., et al., 2005. A freezing-like posture to pictures of mutilation. Psychophysiology 42, 255-260. https://doi.org/10.1111/j.1469-8986. 2005.00287.x.

Bancaud, J., Talairach, J., 1992. Clinical semiology of frontal lobe seizures. Adv. Neurol. 57, 3-58.

Bastos, A.F., Vieira, A.S., Oliveira, J.M., Oliveira, L., Pereira, M.G., Figueira, I., et al., 2016. Stop or move: defensive strategies in humans. Behav. Brain Res. 302, 252-262. https://doi.org/10.1016/j.bbr.2016.01.043.

Benjamin, D.J., Berger, J.O., Johannesson, M., Nosek, B.A., Wagenmakers, E.J., Berk, R. et al., 2018. Redefine statistical significance. Nat. Hum. Behav. 2 (1), 6-10. https:// doi.org/10.1038/s41562-017-0189-z.

Blakemore, R.L., Vuilleumier, P., 2017. An emotional call to action: integrating affective neuroscience in models of motor control. Emotion Rev. 9 (4), 299-309. https:// doi.org/10.1177/1754073916670020.

Blanchard, R.J., Blanchard, D.C., 1971. Defensive reactions in the albino rat. Learn. Motiv. 2, 351-362. https://doi.org/10.1016/0023-9690(71)90016-6.

Blanchard, R.J., Flannelly, K.J., Blanchard, D.C., 1986. Defensive behaviors of laboratory and wild Rattus norvegicus. J. Comp. Psychol. 100, 101-107. https://doi.org/ 10.1037/0735-7036.100.2.101.

Bürkner, P.-C., 2017. brms: an R package for bayesian multilevel models using Stan. J. Stat. Software 80 (1), 1-28. https://doi.org/10.18637/jss.v080.i01.

Bush, G., Luu, P., Posner, M.I., 2000. Cognitive and emotional influences in anterior cingulate cortex. Trends Cognit. Sci. 4, 215-222. https://doi.org/10.1016/S1364 6613(00)01483-2.

Campagnoli, R.R., Krutman, L., Vargas, C.D., Lobo, I., Oliveira, J.M., Oliveira, L., et al., 2015. Preparing to caress: a neural signature of social bonding. Front. Psychol. 6, 16 https://doi.org/10.3389/fpsyg.2015.00016.

Chen, M., Bargh, J.A., 1999. Immediate behavioral predispositions to approach or avoid the stimulus. Pers. Soc. Psychol. Bull. 25, 215-224. https://doi.org/10.1177/ 0146167299025002007.

Chen, G., Taylor, P.A., Cox, R.W., Pessoa, L., 2019a. How to deal with multiplicity in neuroimaging? A case for global calibration. bioRxiv 706747. https://doi.org/ 10.1101/706747.

Chen, G., Xiao, Y., Taylor, P.A., Rajendra, J.K., Riggins, T., Geng, F., et al., 2019b. Handling multiplicity in neuroimaging through bayesian lenses with multilevel modeling. Neuroinformatics 1-31. https://doi.org/10.1007/s12021-018-9409-6.

Coelho, C.M., Lipp, O.V., Marinovic, W., Wallis, G., Riek, S., 2010. Increased corticospinal excitability induced by unpleasant visual stimuli. Neurosci. Lett. 481, 135-138. https://doi.org/10.1016/j.neulet.2010.03.027.

Cohen, M.S., 1997. Parametric analysis of fMRI data using linear systems methods. Neuroimage 6, 93-103. https://doi.org/10.1006/nimg.1997.0278.

Conty, L., Dezecache, G., Hugueville, L., Grèzes, J., 2012. Early binding of gaze, gesture, and emotion: neural time course and correlates. J. Neurosci. 32, 4531-4539. https:// doi.org/10.1523/JNEUROSCI.5636-11.2012.

Cox, R., 1996. AFNI: software for analysis and visualization of functional magnetic resonance neuroimages. Comput. Biomed. Res. 29, 162-173. https://doi.org/ 10.1006/cbmr.1996.0014.

Damasio, A.R., 1999. The Feeling of what Happens: Body and Emotion in the Making of Consciousness.

Darwin, C., 1872. The Expression of the Emotions in Man and Animals. J. Murray, London.

David, N., Cohen, M.X., Newen, A., Bewernick, B.H., Shah, N.J., Fink, G.R., Vogeley, K., 2007. The extrastriate cortex distinguishes between the consequences of one's own and others' behavior. Neuroimage 36, 1004-1014. https://doi.org/10.1016/ j.neuroimage.2007.03.030. de Gelder, B., Snyder, J., Greve, D., Gerard, G., Hadjikhani, N., 2004. Fear fosters flight: a mechanism for fear contagion when perceiving emotion expressed by a whole body. Proc. Natl. Acad. Sci. Unit. States Am. 101, 16701-16706. https://doi.org/10.1073/ pnas.0407042101, 2004.

de Oliveira, L.A., Imbiriba, L.A., Russo, M.M., Nogueira-Campos, A.A., Rodrigues Ede, C., Pereira, M.G., et al., 2012. Preparing to grasp emotionally laden stimuli. PloS One 7, e45235. https://doi.org/10.1371/journal.pone.0045235.

Destrieux, C., Fischl, B., Dale, A., Halgren, E., 2010. Automatic parcellation of human cortical gyri and sulci using standard anatomical nomenclature. Neuroimage 53 (1), 1-15. https://doi.org/10.1016/j.neuroimage.2010.06.010.

Downing, P.E., Jiang, Y., Shuman, M., Kanwisher, N.G., 2001. A cortical area selective for visual processing of the human body. Science 293, 2470-2473. https://doi.org/ 10.1126/science.1063414, 2001.

Facchinetti, L.D., Imbiriba, L.A., Azevedo, T.M., Vargas, C.D., Volchan, E., 2006. Postural modulation induced by pictures depicting prosocial or dangerous contexts. Neurosci. Lett. 410 (1), 52-56. https://doi.org/10.1016/j.neulet.2006.09.063.

Fanselow, M.S., Lester, L.S., 1988. A functional behavioristic approach to aversively motivated behavior: predatory imminence as a determinant of the topography of defensive behavior. In: Bolles, R.C., Beecher, M.D. (Eds.), Evolution and Learning. Lawrence Erlbaum Associates, Inc, Hillsdale, NJ, US, pp. 185-212.

Fernandes Jr., O., Portugal, L.C., Alves, R.C., Campagnoli, R.R., Mocaiber, I., David, I.P., et al., 2013. How you perceive threat determines your behavior. Front. Hum. Neurosci. 7, 632. https://doi.org/10.3389/fnhum.2013.00632.

Fernandes Jr., O., Portugal, L.C.L., Alves, R.C.S., Arruda-Sanchez, T., Rao, A., Volchan, E., et al., 2017. Decoding negative affect personality trait from patterns of brain activation to threat stimuli. Neuroimage 145, 337-345. https://doi.org/10.1016/ j.neuroimage.2015.12.050.

Flaisch, T., Schupp, H.T., Renner, B., Junghofer, M., 2009. Neural systems of visual attention responding to emotional gestures. Neuroimage 45, 1339-1346. https:// doi.org/10.1016/j.neuroimage.2008.12.073.

Frijda, N.H., 1986. Studies in Emotion and Social Interaction. The Emotions. Cambridge University Press; Paris, France: Editions de la Maison des Sciences de l'Homme, New York, NY, US.

Friston, K.J., Frith, C.D., Turner, R., Frackowiak, R.S.J., 1995. Characterizing evoked hemodynamics with fMRI. Neuroimage 2, 157-165. https://doi.org/10.1006/ nimg.1995.1019.

Goodkind, M., Eickhoff, S.B., Oathes, D.J., Jiang, Y., Chang, A., Jones-Hagata, L.B., Ortega, B.N., Zaiko, Y.V., Roach, E.L., Korgaonkar, M.S., Grieve, S.M., GalatzerLevy, I., Fox, P.T., Etkin, A., 2015. Identification of a common neurobiological substrate for mental illness. JAMA Psychiatr. 72, 305-315. https://doi.org/10.1001/ jamapsychiatry.2014.2206.

Grèzes, J., Pichon, S., de Gelder, B., 2007. Perceiving fear in dynamic body expressions. Neuroimage 35, 959-967. https://doi.org/10.1016/j.neuroimage.2006.11.030.

Grèzes, J., Philip, L., Chadwick, M., Dezecache, G., Soussignan, R., Conty, L., 2013. Selfrelevance appraisal influences facial reactions to emotional body expressions. PloS One 8, e55885. https://doi.org/10.1371/journal. pone.0055885.

Grosbras, M.H., Paus, T., 2006. Brain networks involved in viewing angry hands or faces. Cerebr. Cortex 16, 1087-1096. https://doi.org/10.1093/cercor/bhj050.

Hajcak, G., Molnar, C., George, M.S., Bolger, K., Koola, J., Nahas, Z., 2007. Emotion facilitates action: a transcranial magnetic stimulation study of motor cortex excitability during picture viewing. Psychophysiology 44 (1), 91-97. https://doi.org/ 10.1111/j.1469-8986.2006.00487.x.

Kohn, N., Eickhoff, S.B., Scheller, M., Laird, A.R., Fox, P.T., Habel, U., 2014. Neural network of cognitive emotion regulation-an ALE meta-analysis and MACM analysis. Neuroimage 87, 345-355. https://doi.org/10.1016/j.neuroimage.2013.11.001.

Kolesar, T.A., Kornelsen, J., Smith, S.D., 2017. Separating neural activity associated with emotion and implied motion: an fMRI study. Emotion 17 (1), 131-140. https:// doi.org/10.1037/emo0000209.

Kornelsen, J., Smith, S.D., McIver, T.A., 2014. A neural correlate of visceral emotional responses: evidence from fMRI of the thoracic spinal cord. Soc. Cognit. Affect Neurosci. 10, 584-588. https://doi.org/10.1093/scan/nsu092.

Kret, M.E., Pichon, S., Grezes, J., de Gelder, B., 2011. Similarities and differences in perceiving threat from dynamic faces and bodies. An fMRI study. Neuroimage 54, 1755-1762. https://doi.org/10.1016/j.neuroimage.2010.08.012.

Kriegeskorte, N., Simmons, W.K., Bellgowan, P.S., Baker, C.I., 2009. Circular analysis in systems neuroscience: the dangers of double dipping. Nat. Neurosci. 12 (5), 535-540. https://doi.org/10.1038/nn.2303.

Krieglmeyer, R., Deutsch, R., 2010. Comparing measures of approach-avoidance behaviour: the manikin task vs. two versions of the joystick task. Cognit. Emot. 24, 810-828. https://doi.org/10.1080/02699930903047298.

Kruschke, John, 2010. Doing Bayesian Data Analysis: a Tutorial with R, JAGS and Stan. Academic Press, USA.

Kühn, S., Keizer, A., Rombouts, S.A., Hommel, B., 2011. The functional and neural mechanism of action preparation: roles of EBA and FFA in voluntary action control. J. Cognit. Neurosci. 23 (1), 214-220. https://doi.org/10.1162/jocn.2010.21418.

Kveraga, K., Boshyan, J., Adams, R.B., Mote, J., Betz, N., Ward, N., et al., 2015. If it bleeds, it leads: separating threat from mere negativity. Soc. Cognit. Affect Neurosci. 10 (1), 28-35. https://doi.org/10.1093/scan/nsu007.

Lang, P.J., Bradley, M.M., Cuthbert, B.N., 1997. Motivated attention: affect, activation, and action. In: Lang, P.J., Simons, R.F., Balaban, M.T. (Eds.), Attention and Orienting: Sensory and Motivational Processes. Lawrence Erlbaum Associates Publishers, Mahwah, NJ, US, pp. 97-135.

Lang, P.J., Bradley, M.M., Cuthbert, B.N., 2005. International Affective Picture System (IAPS): Affective Ratings of Pictures and Instruction Manual. University of Florida, Gainesville, FL. 
Langner, R., Leiberg, S., Hoffstaedter, F., Eickhoff, S.B., 2018. Towards a human selfregulation system: common and distinct neural signatures of emotional and behavioural control. Neurosci. Biobehav. Rev. 90, 400-410. https://doi.org/ 10.1016/j.neubiorev.2018.04.022.

Limanowski, J., Lutti, A., Blankenburg, F., 2014. The extrastriate body area is involved in illusory limb ownership. Neuroimage 86, 514-524. https://doi.org/10.1016/ j.neuroimage.2013.10.035.

McIver, T.A., Kornelsen, J., Smith, S.D., 2013. Limb-specific emotional modulation of cervical spinal cord neurons. Cognit. Affect Behav. Neurosci. 13, 464-472. https:// doi.org/10.3758/s13415-013-0154-x.

McShane, B.B., Gal, D., Gelman, A., Robert, C., Tackett, J.L., 2017. Abandon statistical significance. Am. Statistician 73, 235-245. https://doi.org/10.1080/ 00031305.2018.1527253.

Meyer, C., Padmala, S., Pessoa, L., 2019. Dynamic threat processing. J. Cognit. Neurosci. 31, 522-542. https://doi.org/10.1162/jocn_a_01363.

Misra, G., Coombes, S.A., 2015. Neuroimaging evidence of motor control and pain processing in the human midcingulate cortex. Cerebr. Cortex 25, 1906-1919. https:// doi.org/10.1093/cercor/bhu001.

Nogueira-Campos, A.A., de Oliveira, L.A., Della-Maggiore, V., Esteves, P.O., Rodrigues Ede, C., Vargas, C.D., 2014. Corticospinal excitability preceding the grasping of emotion-laden stimuli. PloS One 9, e94824. https://doi.org/10.1371/ journal.pone.0094824.

Oliveri, M., Babiloni, C., Filippi, M.M., Caltagirone, C., Babiloni, F., Cicinelli, P., et al., 2003. Influence of the supplementary motor area on primary motor cortex excitability during movements triggered by neutral or emotionally unpleasant visual cues. Exp. Brain Res. 149, 214-221. https://doi.org/10.1007/s00221-002-1346-8.

Orgs, G., Dovern, A., Hagura, N., Haggard, P., Fink, G.R., Weiss, P.H., 2016. Constructing visual perception of body movement with the motor cortex. Cerebr. Cortex 26 (1), 440-449. https://doi.org/10.1093/cercor/bhv262 (New York, N.Y.: 1991).

Peelen, M.V., Atkinson, A.P., Andersson, F., Vuilleumier, P., 2007. Emotional modulation of body-selective visual areas. Soc. Cognit. Affect Neurosci. 2, 274-283. https:// doi.org/10.1093/scan/nsm023.

Pereira, M.G., de Oliveira, L., Erthal, F.S., Joffily, M., Mocaiber, I.F., Volchan, E., Pessoa, L., 2010. Emotion affects action: midcingulate cortex as a pivotal node of interaction between negative emotion and motor signals. Cognit. Affect Behav. Neurosci. 10 (1), 94-106. https://doi.org/10.3758/CABN.10.1.94.

Pessoa, L., 2013. The Cognitive-Emotional Brain: from Interactions to Integration. MIT Press, Cambridge, MA, US.

Phaf, R.H., Mohr, S.E., Rotteveel, M., Wicherts, J.M., 2014. Approach, avoidance, and affect: a meta-analysis of approach-avoidance tendencies in manual reaction time tasks. Front. Psychol. 5, 378. https://doi.org/10.3389/fpsyg.2014.00378.

Picard, N., Strick, P.L., 1996. Motor areas of the medial wall: a review of their location and functional activation. Cerebr. Cortex 6, 342-353. https://doi.org/10.1093/ cercor/6.3.342.

Pichon, S., de Gelder, B., Grèzes, J., 2008. Emotional modulation of visual and motor areas by dynamic body expressions of anger. Soc. Neurosci. 3, 199-212. https:// doi.org/10.1080/17470910701394368.

Pichon, S., de Gelder, B., Grèzes, J., 2009. Two different faces of threat. Comparing the neural systems for recognizing fear and anger in dynamic body expressions. Neuroimage 47, 1873-1883. https://doi.org/10.1016/j.neuroimage.2009.03.084.

Pichon, S., de Gelder, B., Grèzes, J., 2012. Threat prompts defensive brain responses independently of attentional control. Cerebr. Cortex 22, 274-285. https://doi.org/ 10.1093/cercor/bhr060.

Poldrack, R.A., Gorgolewski, K.J., Varoquaux, G., 2019. Computational and informatic advances for reproducible data analysis in neuroimaging. Annu. Rev. Biomed. Data Sci. 119-138. https://doi.org/10.1146/annurev-biodatasci-072018-021237.

Ponseti, J., Bosinski, H.A., Wolff, S., Peller, M., Jansen, O., Mehdorn, H.M., et al., 2006. A functional endophenotype for sexual orientation in humans. In: NeuroImage, vol. 33, pp. 825-833. https://doi.org/10.1016/j.neuroimage.2006.08.002.

Ratner, S.C., 1967. Comparative aspects of hypnosis. In: Gordon, J.E. (Ed.), In Handbook of Clinical and Experimental Hypnosis. Macmillan, New York, pp. 550-587.

Roelofs, K., Minelli, A., Mars, R.B., van Peer, J., Toni, I., 2009. On the neural control of social emotional behavior. Soc. Cognit. Affect Neurosci. 4 (1), 50-58. https:// doi.org/10.1093/scan/nsn036.

Rousselet, G.A., Pernet, C.R., Wilcox, R.R., 2017. Beyond differences in means: robust graphical methods to compare two groups in neuroscience. Eur. J. Neurosci. 46, 1738-1748. https://doi.org/10.1111/ejn.13610.

Saraiva, A.C., Schüür, F., Bestmann, S., 2013. Emotional valence and contextual affordances flexibly shape approach-avoidance movements. Front. Psychol. 4, 933. https://doi.org/10.3389/fpsyg.2013.00933.

Shackman, A.J., Salomons, T.V., Slagter, H.A., Fox, A.S., Winter, J.J., Davidson, R.J., 2011. The integration of negative affect, pain and cognitive control in the cingulate cortex. Nat. Rev. Neurosci. 12 (3), 154-167. https://doi.org/10.1038/nrn2994.
Siegel, J.S., Power, J.D., Dubis, J.W., Vogel, A.C., Church, J.A., Schlaggar, B.L., Petersen, S.E., 2014. Statistical improvements in functional magnetic resonance imaging analyses produced by censoring high motion data points. Hum. Brain Mapp. 35, 1981-1996. https://doi.org/10.1002/hbm.22307.

Simos, P.G., Kavroulakis, E., Maris, T., Papadaki, E., Boursianis, T., Kalaitzakis, G., Savaki, H.E., 2017. Neural foundations of overt and covert actions. Neuroimage 52, 482-496. https://doi.org/10.1016/j.neuroimage.2017.03.036.

Sinke, C.B., Sorger, B., Goebel, R., de Gelder, B., 2010. Tease or threat? Judging social interactions from bodily expressions. Neuroimage 49, 1717-1727. https://doi.org/ 10.1016/j.neuroimage.2009.09.065.

Smith, S.D., Kornelsen, J., 2011. Emotion-dependent responses in spinal cord neurons: a spinal fMRI study. Neuroimage 58 (1), 269-274. https://doi.org/10.1016/ j.neuroimage.2011.06.004.

Stan Development Team, 2016. Stan Modeling Language Users Guide and Reference Manual. Retrieved from. http://mc-stan.org.

Steinmetz, P.N., Cabrales, E., Wilson, M.S., Baker, C.P., Thorp, C.K., Smith, K.A., Treiman, D.M., 2011. Neurons in the human hippocampus and amygdala respond to both low- and high-level image properties. J. Neurophysiol. 105 (6), 2874-2884. https://doi.org/10.1152/jn.00977.2010.

Straube, T., Schmidt, S., Weiss, T., Mentzel, H.J., Miltner, W.H., 2009. Dynamic activation of the anterior cingulate cortex during anticipatory anxiety. Neuroimage 44, 975-981. https://doi.org/10.1016/j.neuroimage.2008.10.022.

Talairach, J., Bancaud, J., Geier, S., Bordas-Ferrer, M., Bonis, A., Szikla, G., Rusu, M., 1973. The cingulate gyrus and human behaviour. Electroencephalogr. Clin. Neurophysiol. 34, 45-52.

Talairach, J., Tournoux, P., 1988. Co-planar Stereotaxic Atlas of the Human Brain. Thieme, New York.

Tomasino, B., Weiss, P.H., Fink, G.R., 2012. Imagined tool-use in near and far space modulates the extra-striate body area. Neuropsychologia 50, 2467-2476. https:// doi.org/10.1016/j.neuropsychologia.2012.06.018.

Urgesi, C., Berlucchi, G., Aglioti, S.M., 2004. Magnetic stimulation of extrastriate body area impairs visual processing of nonfacial body parts. Curr. Biol. 14, 2130-2134. https://doi.org/10.1016/j.cub.2004.11.031.

Van den Stock, J., Tamietto, M., Sorger, B., Pichon, S., Grézes, J., de Gelder, B., 2011. Cortico-subcortical visual, somatosensory, and motor activations for perceiving dynamic whole-body emotional expressions with and without striate cortex (V1). Proc. Natl. Acad. Sci. U.S.A. 108, 16188-16193. https://doi.org/10.1073/ pnas. 1107214108 .

Van den Stock, J., Hortensius, R., Sinke, C., Goebel, R., de Gelder, B., 2015. Personality traits predict brain activation and connectivity when witnessing a violent conflict. Sci. Rep. 5, 13779. https://doi.org/10.1038/srep13779.

Van Loon, A.M., van den Wildenberg, W.P.M., van Stegeren, A.H., Ridderinkhof, K.R., Hajcak, G., 2010. Emotional stimuli modulate readiness for action: a transcranial magnetic stimulation study. Cognit. Affect Behav. Neurosci. 10, 174-181. https:// doi.org/10.3758/CABN.10.2.174.

Vogt, B.A., 2005. Pain and emotion interactions in subregions of the cingulate gyrus. Nat. Rev. Neurosci. 6, 533-544. https://doi.org/10.1038/nrn1704.

Vogt, B.A., 2016. Midcingulate cortex: structure, connections, homologies, functions and diseases. J. Chem. Neuroanat. 74, 28-46. https://doi.org/10.1016/ j.jchemneu.2016.01.010.

Vogt, B.A., Vogt, L.J., 2009. Opioids, placebos and descending control of pain and stress systems. In: Vogt, B.A. (Ed.), Cingulate Neurobiology and Disease. Oxford University Press, London, pp. 339-364.

Vogt, B.A., Berger, G.R., Derbyshire, S.W., 2003. Structural and functional dichotomy of human midcingulate cortex. Eur. J. Neurosci. 18, 3134-3144. https://doi.org/ 10.1111/j.1460-9568.2003.03034.x.

Vogt, B.A., Vogt, L., Laureys, S., 2006. Cytology and functionally correlated circuits of human posterior cingulate areas. Neuroimage 29, 452-466. https://doi.org/ 10.1016/j.neuroimage.2005.07.048.

Volchan, E., Souza, G.G., Franklin, C.M., Norte, C.E., Rocha-Rego, V., Oliveira, J.M., et al., 2011. Is there tonic immobility in humans? Biological evidence from victims of traumatic stress. Biol. Psychol. 88 (1), 13-19. https://doi.org/10.1016/ j.biopsycho.2011.06.002.

Volchan, E., Rocha-Rego, V., Bastos, A.F., Oliveira, J.M., Franklin, C., Gleiser, S., et al., 2017. Immobility reactions under threat: a contribution to human defensive cascade and PTSD. Neurosci. Biobehav. Rev. 76, 29-38. https://doi.org/10.1016/ j.neubiorev.2017.01.025.

Zimmermann, M., Mars, R.B., de Lange, F.P., Toni, I., Verhagen, L., 2017. Is the extrastriate body area part of the dorsal visuomotor stream? Brain Struct. Funct. 223 (1), 31-46. https://doi.org/10.1007/s00429-017-1469-0. 